\begin{tabular}{|c|ccc|c|}
\hline PORT SAID ENGINEERING RESEARCH JOURNAL \\
\hline Vaculty of Engineering - Port Said University \\
No. 2 & September 2018 & pp: $85: 101$
\end{tabular}

\title{
CFD Simulation and Losses Analysis of a Beta-Type Stirling Engine
}

\author{
N. N. Mikhael $^{1}$, M.El-Ghandou ${ }^{2}$, S. A. El-Ghafour ${ }^{3}$
}

\begin{abstract}
A three-dimensional Computational Fluid Dynamics (CFD) simulation for the beta-type Stirling engine is performed. Firstly, a thorough characterization of the thermal and fluid flow fields during the cycle is presented. Secondly, a comprehensive energy analysis for the engine is conducted to accurately identify the sources and magnitudes of thermodynamic losses. Computational results show a close agreement with the experimental results with an accuracy of about $96 \%$. Within the compression and expansion spaces, the dominant heat transfer rates occur during the expansion strokes due to the significant impinging effect of the tumble vortices generated from the flow jetting. Furthermore, the jetting and ejecting processes into the regenerator is characterized by a significant temperature gradient and a large matrix temperature oscillation. The pressure difference between the expansion and compression spaces is the main driver for the flow leakage through the appendix gap. From the energy analysis, the regenerator thermal loss and the pumping power represent the largest part of the Stirling engine losses by about $9.2 \%$ and $7.5 \%$, respectively.
\end{abstract}

Keywords: Beta-type Stirling engine, GPU-3, CFD, Losses

\section{Introduction}

Fossil fuel still dominates the global energy market. According to the U.S. Energy Information Administration (EIA), fossil fuel supplies $80 \%$ of the world's current energy [1]. However, the massive shortage of the fossil fuel resources, the fluctuations in fuel prices, and the severe environmental problems due to its combustion inevitably lead to reduce our dependence on this type of energy source and widening use of renewable energy resources. In this regard, Stirling engine has especially emerged as a promising option among numerous power generation applications. Its ability of being powered using a wide variety of renewable resources such as solar, geothermal and biomass [2], besides the conventional heat sources, gives Stirling engine a high level of reliability.

For a long time, the difficulty of modeling the real Stirling engine was one of the most common reasons of its precluding. The highly transient phenomena and the complexity of the losses mechanisms occurring within this engine are considered challenges facing any trial for accurate simulation. According to the degree of complexity, Martini [3] classified the models of Stirling engine as first-, second- and third-orders. Later, another category was added to Martini's classification, namely

\footnotetext{
${ }^{1}$ Emirate Professor, MPE Department, Faculty of Engineering, PortSaid University, Port-Said, Egypt,Email:nadymikhael@gmail.com

${ }^{2}$ Assistant Professor, MPE Department, Faculty of Engineering, PortSaid University, Port-Said, Egypt, Email: melghandourel@gmail.com

${ }^{3}$ Assistant lecturer, MPE Department, Faculty of Engineering, Port-

Said University, Port-Said, Egypt,Email: s.a.ghafour@eng.psu.edu.eg
}

fourth-order models. The first-order models are simple analytical analyses which are usually used for the thermodynamic simulation of Stirling cycles, instead of simulating real engines [4]. Schmidt model, also called isothermal model, is one of the most common attempts under this type. In 1960, the adiabatic working spaces analysis, conducted by Finkelstein, substituted for the isothermal one [5]. Later, Urieli and Berchowitz further modified the analysis of the adiabatic model by implementing the effects of the imperfect processes within the engine, which is known as "Simple model" [6]. This model has become the base for the secondorder models. In this type of models, the engine volume is usually discretized into five cells (heater, regenerator, cooler, expansion and compression spaces). The ordinary differential form of the mass and energy conservation equations are applied to each cell considering the transient nature of the flow. Over the past decade, efforts have been made to improve the accuracy of the secondorder models. This is achieved by better characterization of the engine irreversibilities and losses [4, 7-11]. The third-order model, sometimes named nodal model, is another type of Stirling engine analysis. It is more complex than the second-order one and needs much more intensive numerical calculations. As, the engine volume is discretized into several control volumes of various sizes and shapes [12]. The partial differential form of conservation of mass, energy and momentum together with the equation of state of the working gas are applied for each control volume. Accordingly, the thirdorder analysis has reasonably succeeded in describing the behavior of the gas dynamics inside the engine. 
Moreover, fewer assumptions about the thermodynamic cycle and the coupling of loss mechanisms to the global analysis are made [13]. Finkelstein [14], Urieli et al. [15] and Tew [16] are the most famous models belonging to this category. These models have been the base for the subsequent models which incorporated enhanced features to achieve more accurate ones [17, 18].

Although the second- and third-order models have achieved acceptable range of accuracy in Stirling engine modeling, there are some constraints that prevent achieving higher degrees of accuracy. The dependence on steady unidirectional flow rather than unsteady flow correlations, for heat transfer and fluid friction encountered in heat exchangers, may lead to the discrepancy in results [19]. Furthermore, the rates of heat transfer in compression and expansion spaces cannot be accurately predicted by the assumption of uniform temperature distribution and constant heat transfer coefficients which are usually adopted by several models [20]. More importantly, the flow maldistribution in engine components, sudden change of flow area, flow recirculation and other important features cannot be predicted using these zero- or one-dimensional models. Such features directly affect the whole engine performance [21]. All these issues supported the use of the multidimensional multiphysics Computational Fluid Dynamics (CFD) modeling, the so-called fourth-order models. Initially, the researchers developed specific twodimensional CFD codes for modeling Stirling engine components, such as piston-cylinder arrangement and regenerator [22, 23]. However, the difficulty of exact specification of the oscillating artificial boundary conditions weakened the confidence in the results of this method. Unfortunately, the initial attempts at whole engine simulation ended in unconverged solutions [24, 25]. The pioneer researchers who came up with a fully converged solution of the whole Stirling engine, Mahkmov and Ingham [26], conducted an axisymmetric simulation of a solar Stirling engine. They presented the complexity of the fluid flow in the engine components. This, consequently, resulted in an accurate prediction of the engine performance compared with the second-order results. The validation of their CFD results was made afterwards [27], indicating an error in the power prediction of approximately $40 \%$. On the other side, Ibrahim et al. [28] proposed another technique in CFD modeling of Stirling engine. As a preliminary step, they conducted the 2D simulation of each component in a free-piston Stirling engine separately. After reaching steady state results with good energy balance for each component, they integrated the whole engine. The researchers affirmed that this approach proved to be successful in cutting down the number of iterations as well as the number of cycles to reach a steady oscillatory solution. With the increasing recommendation of the three-dimensional modeling of Stirling engine, Mahkmov [29] succeeded for a first time in performing a 3D CFD simulation to a $\gamma$-type Stirling engine. The predictions were about $12-18 \%$ different from the experimental data compared with $30 \%$ for the secondorder results. Recently, both research conducted by Salazar and Chen [20] and Chen et al. [30] on a simple $\beta$-type and $\gamma$-type, respectively, characterized the complex heat transfer mechanisms inside the engine. In the most recent CFD investigation, by Alfarawi et al. [31], the maximum deviation in predicting the indicated power of a $\gamma$-type Stirling engine was about $9 \%$ compared to experimental results. The recirculation and vortex separation of velocity streamlines in the engine dead volumes were shown, indicating the reasons of the reduction in output power.

From the above survey, one can observe that the multidimensional CFD analysis emerges as a more accurate technique for Stirling engine modeling compared with the second- and third-order analyses [27]. This is, besides, the successfulness of this technique in depiction and explanation of the real phenomena occurred in Stirling engine. Nevertheless, the phenomena related to the losses mechanisms are still uncovered. This is despite of the expected capability of a better characterization for these losses with the help of CFD simulations. Generally, the losses of Stirling engine can be mainly classified as flow, thermal and mechanical losses. The flow losses include the pumping, the power loss due to piston finite speed and the leakage from the piston seal. Whereas, hysteresis loss, regenerator heat loss, shuttle heat transfer by the displacer and enthalpy pumping losses through the appendix gap as well as heat conduction loss through the regenerator and the cylinder walls are classified as the thermal ones.

The current study firstly presents a comprehensive characterization of the thermal and fluid flow fields during the cycle. Secondly, the effects of the unsteady behaviors of both thermodynamic and fluid dynamic on the heat transfer phenomena and, consequently, the losses mechanisms are investigated. Finally, a thorough energy analysis of the engine is conducted to accurately identify the magnitudes of the different losses. Here, a three-dimensional CFD simulation is performed on a $\beta$ type Stirling engine, namely Ground Power Unit-3 (GPU-3).

\section{Description of Engine Geometry and Operating Conditions}

One of the most famous Stirling engines of $\beta$-type is the Ground Power Unit-3 (GPU-3) engine. An isometric view with the main components of the GPU-3 Stirling engine is illustrated in Fig. 1. 
The main components of the engine include: cylinder, power piston, displacer piston, heater, regenerator, cooler, cooler-end connection and rhombic drive mechanism. The working space within the cylinder is divided into expansion and compression spaces which are separated by the displacer. The design specifications of the engine and the operating condition of the baseline case are summarized in Table 1. Further details of the engine description and specifications have been documented in $[3,32]$. The rhombic drive mechanism, a special mechanism for the $\beta$-type Stirling engine, is also shown in Fig. 1. This mechanism does not produce sinusoidal volume variations. Hence, the exact functions of compression and expansion volumes are derived from kinematics of the drive mechanism and cylinder geometric parameters, as displayed in Fig. 2.

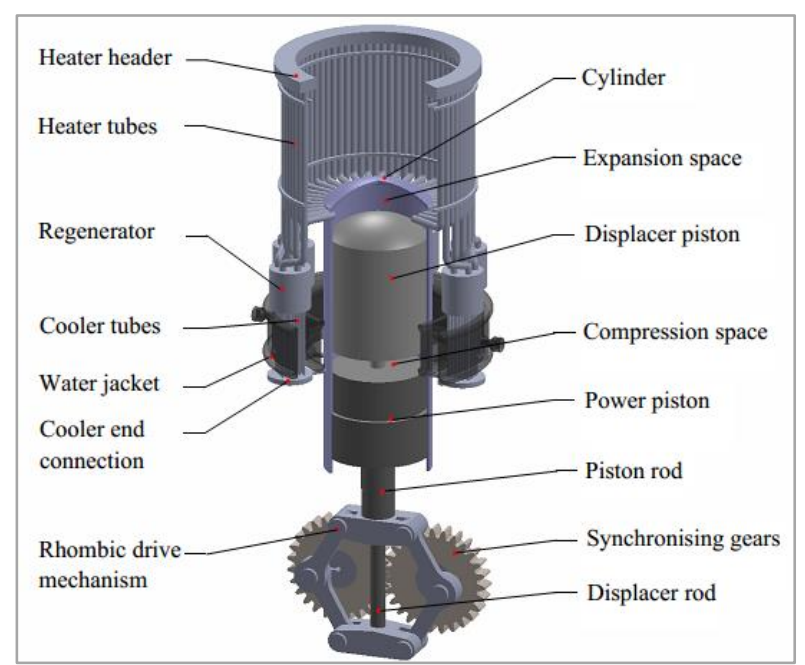

Fig. 1 Isometric view of the GPU - 3 Stirling engine.

From the above figure, the displacement of the piston $y_{p}(\theta)$ and displacer $y_{d}(\theta)$ as a function of crank angle $(\theta)$ can be deduced as follows:

$$
\begin{aligned}
& y_{p}(\theta)=R_{c} \sin (\theta)+\sqrt{\left[L^{2}-\left(e+R_{c} \cos (\theta)\right)^{2}\right]}+L_{p c r}+L_{p} \\
& y_{d}(\theta)=R_{c} \sin (\theta)-\sqrt{\left[L^{2}-\left(e+R_{c} \cos (\theta)\right)^{2}\right]}+L_{d c r}+L_{d}
\end{aligned}
$$

The instantaneous velocities of the piston and displacer, $v_{p}(\theta)$ and $v_{d}(\theta)$, are then calculated by respectively differentiating Eqs. (1) and (2):

$\mathrm{v}_{\mathrm{p}}(\theta)=$

$\omega_{\mathrm{r}} \mathrm{R}_{\mathrm{c}}\left\{\cos (\theta)+\sin (\theta)\left(\mathrm{e}+\mathrm{R}_{\mathrm{c}} \cos (\theta)\right) / \sqrt{\left[\mathrm{L}^{2}-\left(\mathrm{e}+\mathrm{R}_{\mathrm{c}} \cos (\theta)\right)^{2}\right]}\right\}$

(3)

$\mathrm{v}_{\mathrm{d}}(\theta)=$

$\omega_{\mathrm{r}} \mathrm{R}_{\mathrm{c}}\left\{\cos (\theta)-\sin (\theta)\left(\mathrm{e}+\mathrm{R}_{\mathrm{c}} \cos (\theta)\right) / \sqrt{\left[\mathrm{L}^{2}-\left(\mathrm{e}+\mathrm{R}_{\mathrm{c}} \cos (\theta)\right)^{2}\right]}\right\}$

(4)

where $\omega r$ is the angular frequency, $\mathrm{rad} / \mathrm{s}$. Then, the actual volume variations of compression and expansion spaces, $\mathrm{V}_{\mathrm{c}}(\theta)$ and $\mathrm{V}_{\mathrm{e}}(\theta)$, respectively, are functions of the various geometric parameters of the engine and can be further written as:
$\mathrm{V}_{\mathrm{c}}(\theta)=\frac{\pi}{4} \mathrm{~d}_{\mathrm{b}}^{2}\left(\mathrm{y}_{\mathrm{d}}(\theta)-\mathrm{L}_{\mathrm{d}}-\mathrm{y}_{\mathrm{p}}(\theta)\right)$

$\mathrm{V}_{\mathrm{e}}(\theta)=\frac{\pi}{4} \mathrm{~d}_{\mathrm{b}}^{2}\left(\mathrm{~L}_{\mathrm{tot}}-\mathrm{y}_{\mathrm{d}}(\theta)\right)$

where $d_{b}$ is the mean bore of the cylinder. It should be noted that the current rhombic dive mechanism was designed with links of the same length, $\mathrm{L}$.

\section{Governing Equations}

In the present research, the flow is oscillatory, viscous, compressible and Newtonian. Considering a Cartesian coordinate system and decomposing the flow variables (velocity, temperature and pressure), into an ensembleaveraged values, $u, T$ and $p$ and fluctuation, $u^{\prime}, T^{\prime}$ and $p^{\prime}$, the Unsteady Reynolds-Averaged Navier-Stokes (URANS) equations can be yielded, in tensor notation as:

Equation for conservation of mass:

$$
\frac{\partial \rho}{\partial \mathrm{t}}+\frac{\partial}{\partial \mathrm{x}_{\mathrm{i}}}\left(\rho \mathrm{u}_{\mathrm{r}_{\mathrm{i}}}\right)=0
$$

Equations for conservation of momentum:

$$
\begin{gathered}
\frac{\partial\left(\rho \cdot \mathrm{u}_{i}\right)}{\partial \mathrm{t}}+\frac{\partial\left(\rho \cdot \mathrm{u}_{r_{j}} \cdot \mathrm{u}_{i}\right)}{\partial \mathrm{x}_{j}}=-\frac{\partial \mathrm{p}}{\partial \mathrm{x}_{i}}+\frac{\partial}{\partial \mathrm{x}_{j}}\left[\mu \cdot\left(\frac{\partial \mathrm{u}_{i}}{\partial \mathrm{x}_{j}}+\frac{\partial \mathrm{u}_{j}}{\partial \mathrm{x}_{i}}\right)+\right. \\
\left.\lambda \cdot \delta_{i j} \cdot \frac{\partial \mathrm{u}_{k}}{\partial \mathrm{x}_{k}}-\rho \cdot \overline{\mathrm{u}_{l} \cdot \mathrm{u}_{\mathrm{j}}}\right]
\end{gathered}
$$

where $\rho, \mu$ and $\lambda$ are the density, the dynamic viscosity and the bulk viscosity coefficient, respectively. According to the Stokes hypothesis, $\lambda$ can be written as [33]:

$$
\lambda=-\frac{2}{3} \mu
$$

and $\delta_{\mathrm{ij}}$ is Kronecker-Delta operator which either equals one if $\mathrm{i}=\mathrm{j}$ or zero if $\mathrm{i} \neq \mathrm{j}$.

The Boussinesq approximation essentially assumed that the Reynolds stresses $\left(-\rho . \overline{u_{l}}\right.$. ú $)$ can be modelled using the turbulent viscosity, $\mu_{t}$, which is analogous to molecular viscosity. Thus, the approximation is [34]:

$-\rho \cdot \overline{\mathrm{u}_{\mathrm{r}} \cdot \mathrm{u}_{\mathrm{j}}} \approx \mu_{\mathrm{t}} \cdot\left[\left(\frac{\partial \mathrm{u}_{\mathrm{i}}}{\partial \mathrm{x}_{\mathrm{j}}}+\frac{\partial \mathrm{u}_{\mathrm{j}}}{\partial \mathrm{x}_{\mathrm{i}}}\right)-\frac{2}{3} \cdot \delta_{\mathrm{ij}} \cdot \frac{\partial \mathrm{u}_{\mathrm{k}}}{\partial \mathrm{x}_{\mathrm{k}}}\right]-\frac{2}{3} \cdot \rho \cdot \mathrm{k} \cdot \delta_{\mathrm{ij}}(\mathbf{1 0})$ where $\mathrm{k}$ is turbulent kinetic energy.

Equation for conservation of energy:

$$
\begin{gathered}
\frac{\partial(\rho \cdot E)}{\partial \mathrm{t}}+\frac{\partial}{\partial \mathrm{x}_{\mathrm{i}}}\left[\mathrm{u}_{\mathrm{r}_{\mathrm{i}}}(\rho \cdot \mathrm{E}+\mathrm{p})\right]=\frac{\partial}{\partial \mathrm{x}_{\mathrm{i}}}\left[\mathrm{K}_{\mathrm{eff}} \cdot \frac{\partial \mathrm{T}}{\partial \mathrm{x}_{\mathrm{i}}}-\right. \\
\left.\rho \cdot \mathrm{C}_{\mathrm{p}} \cdot \overline{\mathrm{u}_{\mathrm{r}} \cdot \dot{\mathrm{T}}}\right]+\varphi
\end{gathered}
$$

where $\varphi$ and $\mathrm{E}$ are the viscous dissipation term and the specific energy, respectively, while $\mathrm{K}_{\mathrm{eff}}$ is the effective thermal conductivity, defined as:

$$
\mathrm{K}_{\text {eff }}=\mathrm{K}+\mathrm{K}_{\mathrm{t}}
$$

where $\mathrm{K}$ and $\mathrm{K}_{\mathrm{t}}$ is the laminar and turbulent thermal conductivity, respectively. Also, $\overline{u_{1}} \dot{T}$ is the turbulent heat flux term and can be assumed as [34]:

$$
\overline{\mathrm{u}_{l} \cdot \grave{T}}=-\alpha_{\mathrm{t}} \cdot \frac{\partial \mathrm{T}}{\partial \mathrm{x}_{\mathrm{i}}}
$$


where $\alpha_{t}$ is the turbulent diffusivity and usually related to eddy viscosity via a turbulent Prandtl number, $\operatorname{Pr}_{t}$, [33]:

$$
\operatorname{Pr}_{\mathrm{t}}=\frac{\mu_{\mathrm{t}}}{\rho \cdot \alpha_{\mathrm{t}}} \approx 0.85-0.9
$$

Table 1 Design specifications and operating condition of the GPU-3 Stirling engine, units in $\mathrm{cm}$ [3].

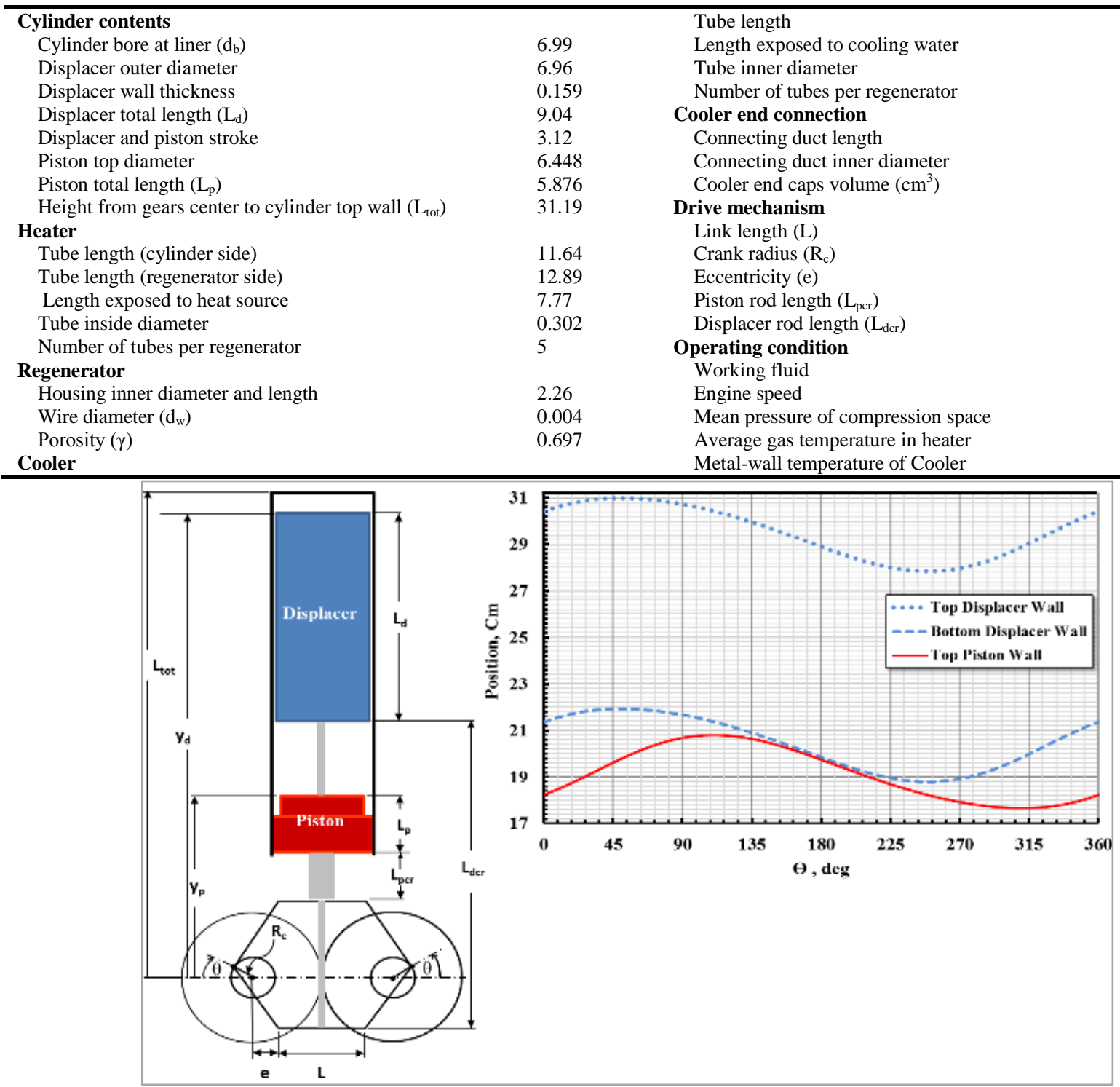

Fig. 2 Kinematic analysis of the rhombic drive mechanism: (a) dimension definitions and (b) displacement variations of piston and displacer versus crank angle.

In the above equations, $u_{r}$ is the relative velocity between fluid and local moving frame [30]. This relative velocity is applied in zones that are deformed due to the movement of piston and displacer. Here, it is worth mentioning that the effect of viscous dissipation term, $\varphi$, on the temperature in Eq. (11) can be ignored compared with the effect of heat transfer and compression during the cycle [29]. Also, the turbulent viscosity, $\mu_{\mathrm{t}}$, is defined according to the URANS model being used. Among the different models, the realizable $\mathrm{k}-\varepsilon$ model with enhanced wall treatment produces the most accurate predictions for engine performance [32]. Hence it is used in the current study. The equations of this model can be found in Ref. [32].

\section{Equations of State}

The working fluid is considered ideal gas. Therefore, it obeys the equation of state:

$$
\mathrm{p} \cdot \mathrm{V}=\mathrm{m} \cdot \mathrm{R} \cdot \mathrm{T}
$$

where $\mathrm{m}$ and $\mathrm{R}$ are the mass and the gas constant, respectively.

\subsection{Regenerator Equations}

In the multi-D analysis, the regenerator is frequently modelled using the porous media approach [20], [28] [30], [31]. The main physical phenomena to be considered in the characterization of the regenerator are the flow friction and the heat transfer [35].

\subsubsection{Flow modeling in porous media}

The flow through the porous media is modeled by the addition of a source term, $\mathrm{S}$, to the momentum 
equations in each of the three coordinate directions (x, $\mathrm{y}$ and $\mathrm{z})$ as follows [33]:

$$
\mathrm{S}_{\mathrm{i}}=-\left(\frac{\mu}{\alpha_{r}} \mathrm{u}_{\mathrm{i}}+\mathrm{C}_{2} \frac{1}{2} \rho \mathrm{u}_{\mathrm{i}} \cdot\left|\mathrm{u}_{\mathrm{i}}\right|\right)
$$

where $1 / \alpha_{r}$ and $C_{2}$ are the viscous and inertial resistance coefficients, respectively. These coefficients are hydrodynamic characteristics linked to the structure of the porous media. These parameters can be generally deduced from a polynomial curve which adjusts the data points representing the flow friction coefficient vs. Reynolds number through the regenerator. The standard two-parameter Ergun form [36] is currently selected to correlate the friction coefficient, $\mathrm{C}_{\mathrm{f}}$, with Reynolds number, as follows:

$$
\mathrm{C}_{\mathrm{f}}=\frac{\mathrm{a}_{1}}{\mathrm{Re}}+\mathrm{a}_{2}
$$

According to this correlation, the coefficients can be written as:

$$
\begin{gathered}
\frac{1}{\alpha_{\mathrm{r}}}=\frac{1}{2} \mathrm{a}_{1} \frac{1}{\gamma \mathrm{d}_{\mathrm{h}}^{2}} \\
\mathrm{C}_{2}=\frac{\mathrm{a}_{2}}{\gamma^{2} \mathrm{~d}_{\mathrm{h}}}
\end{gathered}
$$

where $\gamma$ and $d_{h}$ denote porosity and hydraulic diameter of the matrix, respectively. The hydraulic diameter is related to the wire diameter $\left(\mathrm{d}_{\mathrm{w}}\right)$ according to Walker and Vasishta [37] as:

$$
\mathrm{d}_{\mathrm{h}}=\frac{\gamma \mathrm{d}_{\mathrm{w}}}{(1-\gamma)}
$$

and $\operatorname{Re}$ is obtained by:

$$
\operatorname{Re}=\frac{\rho \mathrm{ud}_{\mathrm{h}}}{\gamma \mu_{\mathrm{f}}}
$$

where $\mu_{\mathrm{f}}$ is the viscosity of the working fluid.

\subsubsection{Heat Transfer modeling in porous media}

In the current study, the local thermal nonequilibrium approach is selected to model the heat transfer within regenerator. Hence, a dual cell approach for solid matrix and fluid flow are assumed [33]. This requires additional inputs to account for the convective mode of energy transfer between the two phases, appear as extra source terms in fluid and matrix energy equations.

Equation for conservation of energy in Fluid zone:

$$
\begin{gathered}
\frac{\partial}{\partial \mathrm{t}}\left(\gamma \cdot \rho_{\mathrm{f}} \cdot \mathrm{E}_{\mathrm{f}}\right)+\frac{\partial}{\partial \mathrm{x}_{i}}\left[u_{i}\left(\rho_{\mathrm{f}} \cdot \mathrm{E}_{\mathrm{f}}+\mathrm{p}\right)\right]=\frac{\partial}{\partial \mathrm{x}_{i}}\left[\gamma \cdot \mathrm{K}_{\mathrm{f}} \cdot \frac{\partial \mathrm{T}_{\mathrm{f}}}{\partial \mathrm{x}_{i}}\right]+\varphi+ \\
\mathrm{h}_{\mathrm{fs} \cdot} \cdot \mathrm{A}_{\mathrm{fs} \cdot} \cdot\left(\mathrm{T}_{\mathrm{f}}-\mathrm{T}_{\mathrm{s}}\right)
\end{gathered}
$$

Equation for conservation of energy for solid matrix:

$$
\begin{aligned}
& \frac{\partial}{\partial \mathrm{t}}\left[(1-\gamma) \cdot \rho_{\mathrm{s}} \cdot \mathrm{E}_{\mathrm{s}}\right]= \\
& \frac{\partial}{\partial \mathrm{x}_{i}}\left[(1-\gamma) \cdot \mathrm{K}_{\mathrm{s}} \cdot \frac{\partial \mathrm{T}_{\mathrm{s}}}{\partial \mathrm{x}_{i}}\right]+\quad \mathrm{h}_{\mathrm{fs}} \cdot \mathrm{A}_{\mathrm{fs}} \cdot\left(\mathrm{T}_{\mathrm{s}}-\mathrm{T}_{\mathrm{f}}\right)
\end{aligned}
$$

where $h_{f s}$ is the heat transfer coefficient for the fluid/solid interface and $\mathrm{A}_{\mathrm{fs}}$ is the interfacial area density. Here, it should be noted that the fluid thermal conductivity, $\mathrm{K}_{\mathrm{f}}$, includes the turbulent contribution
$\left(\mathrm{K}_{\mathrm{t}}\right)$. Generally, the heat transfer coefficient is defined by:

$$
\mathrm{h}_{\mathrm{fs}}=\frac{\mathrm{Nu} \mathrm{K}}{\mathrm{d}_{\mathrm{h}}}
$$

A two-coefficient equation form can be used to correlate Nusselt number, $\mathrm{Nu}$, with Reynolds number as:

$$
\mathrm{Nu}=\mathrm{a}_{3} \mathrm{Re}+\mathrm{a}_{4}
$$

The interfacial area density $\left(\mathrm{A}_{\mathrm{fs}}\right)$ can be derived as:

$$
\mathrm{A}_{\mathrm{fs}}=\frac{4 \gamma}{\mathrm{d}_{\mathrm{h}}}
$$

\section{Methodology of CFD Simulation}

The present simulation is implemented using the commercial CFD code ANSYS FLUENT, v14.5. The computational methodology incorporated in this simulation is explained in the following subsections.

\subsection{Computational Domain and Grid}

As illustrated in Fig.1, the engine geometry is perfectly symmetrical around the cylinder axis. Therefore, just one-eighth of the geometry is used as the computational domain in this simulation, to reduce the computational cost. The domain includes the compression and expansion spaces, appendix gap, heater, regenerator, cooler and cooler-end connection in addition to the displacer material. The wall thickness for each part, except for displacer, is not taken into consideration. The configuration of the computational domain is displayed in Fig. 3.

The figure presents the original layout of the engine at the instance of zero crank angle $(\theta=0)$. The CAD geometry and grid of the domain is yielded using ANSYS DesignModeller and ICEM (V14.5), respectively. Because of the complex geometry, the computational domain is decomposed into 13 different sub-volumes with different topology. Each of them is separately meshed. The connectivity of the various sub-volumes is ensured by means of arbitrary interfaces that connect the faces of the adjacent volumes. A sliding or static interface is used according to the type of each adjacent sub-volumes, from being deforming, rigid body motion or static zones. Conformal interfaces are performed as possible to reduce the interpolation error [38]. 


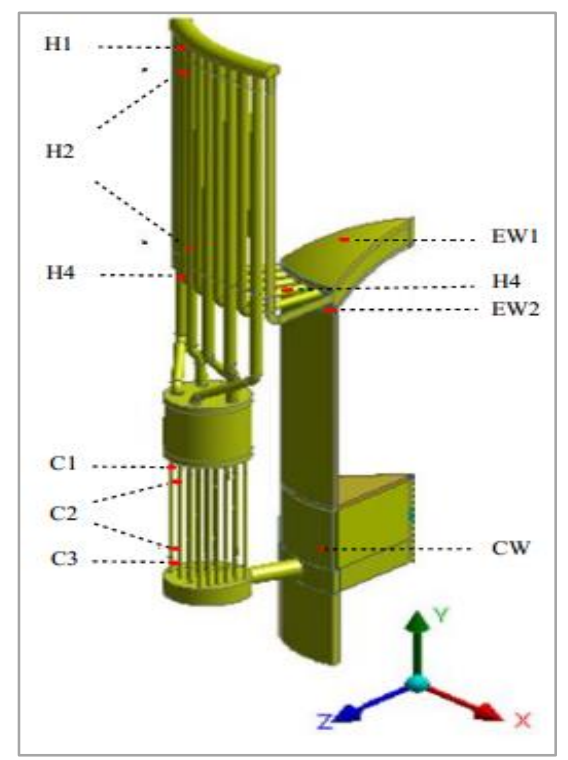

Fig.3 Computational domain.

During the meshing step, some aspects has been followed to permit the applicability of realizable k- $\varepsilon$ turbulence model as well as improve the solution accuracy and convergence characteristics. Firstly, hexahedral elements are used to develop the grid. In fact, it is a big challenge to create a mesh of hexahedral cells in complex geometries than the tetrahedral cells. However, the ability of decreasing the risk of numerical diffusion as well as the demand of computational memory [33], makes it more suitable for the current case. Secondly, a careful refinement is created for regions where large gradients of flow variables are expected, such as regions of area change and near the wall boundaries as well as around sharp corners and curves. In particular, the boundary layer should be covered by nearly 10 cells in the normal direction with $\mathrm{y}^{+} \approx 1$ as possible. This is a recommendation for low-Re grid generation and accurate prediction of heat transfer phenomena [33], [39]. This refinement is performed for all engine walls except for the moving ones. This is attributed to that the moving boundaries need an adaptive refinement technique which largely increases the computational time. Thirdly, to capture the conjugate heat transfer physics at the outside walls of the displacer, the mesh is properly condensed at the fluid/solid interface on both sides. Regarding to the special topology of the regenerator, which consists of dual coincident zones one for the solid matrix and another for fluid, two spatially coincident grids are employed. Also, the two ends of the regenerator grids should be conveniently refined to accurately capture the effect of flow jetting into the regenerator. Finally, the transition from small to large elements should be smooth so that there are no abrupt changes in the size of the grid cells. Areas of low interest could have relatively large elements to keep the total number of elements as minimal as possible.

\subsection{Boundary and Initial Conditions}

Since, only one-eighth of the physical domain is simulated, therefore, periodic boundary conditions are applied to the faces in the azimuthal direction for this sector. No-slip boundary conditions are assigned to all stationary and moving walls. The interior walls of the displacer are treated as adiabatic, while the piston walls are assumed to be maintained at $305 \mathrm{~K}$. It is worth mentioning that the temperature of the walls of heater tubes are not directly specified in the operating condition, as shown in Table 1. Hence, this temperature has been obtained by iteration to achieve two aspects. The first one is to yield an average cyclic gas temperature in the whole tubes of about $950 \mathrm{~K}$. Secondly, the heat added to the engine should only be throughout the length exposed to heat source, whereas a nearly zero cyclic heat transfer rate through the remaining length of the tubes is maintained. In the same manner, the temperatures of cooler walls are imposed. The high thermal inertia of the solid material compared with that of the gas allows for the assumption of isothermal wall boundary temperature for the expansion and compression spaces. The assumed wall temperature is approximately equal to the average cyclic gas temperature in each space. The representation and values of the assumed temperatures of heater, cooler, expansion and compression space walls that applied in the current simulation are illustrated in Table 2 and Fig. 3. A linear temperature distribution is applied for the walls of the other parts .

The initial pressure is selected after several iterations, and it is slightly higher than the minimum pressure of the cycle. For the current calculation, the cycle starts at $\theta=0$ and a pressure of $5.5 \mathrm{MPa}$ is used as an initial pressure.

Table 2 Temperature values of different walls of GPU3 engine.

\begin{tabular}{cc}
\hline Wall Name & Temperature $(\mathbf{K})$ \\
\hline EW1 & 870 \\
EW2 & 805 \\
CW & 325 \\
H1 & 995 \\
H2 & 1090 \\
H3 & 1025 \\
H4 & 975 \\
C1 & 315 \\
C2 & 293 \\
C3 & 307 \\
\hline
\end{tabular}

\subsection{Solution Scheme}

The governing equations are discretized and solved sequentially using FLUENT, a finite volume-based solver. Transient, pressure-based, segregated SIMPLEC solver with absolute velocity formulation 
are the settings for the solution of current simulation. Thermophysical properties (viscosity, specific heat and thermal conductivity) of the working gas are taken to be temperature dependent. The least squares method has been employed for computing the gradients of the transport quantities on the faces of the cell boundaries. The other spatial discretization methods in the simulations are standard for pressure term, and secondorder upwind for continuity, momentum, energy, and flow models transport equations. The explicit relaxation factors of pressure and momentum is set as 0.75 , whereas, the under-relaxation number of 0.9 are applied for density, energy and turbulence quantities. First-order implicit formulation is applied for discretizing the temporal derivatives. All the flow models are used with all default settings given in FLUENT 14.5.

As a technique for speeding up the convergence of the calculation, the simulation is initialized with steady state solution with no piston and displacer motion but with heat transfer calculations being made. Later, the case becomes transient with grid deformation due to motion of the power piston and displacer. The starting with standard $\mathrm{k}-\varepsilon$ turbulence model, local thermal equilibrium porous media approach are needed to meet the convergence criteria during the first cycle of transient solution.

The reciprocating motion of piston and displacer and, consequently, the moving and deforming volumes are simulated using the dynamic mesh technique. User Defined Functions (UDFs) are written and hooked to FLUENT to guide the motion of piston and displacer; according to Eqs. $(3,4)$. Among the different methods of mesh deformation, the grid layering is used to avoid skewed cells when the displacer is at the Top Dead Center (TDC) in the expansion space [38] or when the compression space is fully compressed.

The porous media model requires the input of flow resistance and heat transfer coefficients which are empirically obtained based on experimental results of Tew et al. [40]. They presented the experimental data of the regenerator of GPU-3 in the form of friction factor and Nusselt number as functions of Re. By fitting these data in the form of Eqs. $(17,25)$, the resistance coefficients, $1 / \alpha_{\mathrm{r}}$ and $\mathrm{C}_{\mathrm{f}}$, as well as the Nusselt correlation constants, $\mathrm{a}_{3}$ and $\mathrm{a}_{4}$, can be obtained. The variable heat transfer coefficient between the fluid and the matrix is defined through a UDF using Eqs. (24, 25). Table 3 summarizes the resistance coefficients, interfacial area density and Nusselt correlations for the regenerator of GPU-3 Stirling engine.
Table 3 The resistance coefficients, interfacial area density and Nusselt correlations for the regenerator of GPU-3 engine.

\begin{tabular}{lcccc}
\hline $\mathbf{1 / \alpha}\left(\mathbf{1} / \mathbf{m}^{\mathbf{2}}\right)$ & $\mathbf{C}_{2}(\mathbf{1} / \mathbf{m})$ & $\mathbf{A}_{\mathbf{f s}}(\mathbf{1} / \mathbf{m})$ & $\mathbf{N u}(-)$ \\
\hline $6.0183 \times 10^{+9}$ & $1.2702 \times 10^{+4}$ & \multirow{2}{*}{$30,304.4341$} & If $\mathrm{Re}<25$ & $\mathrm{Nu}=0.2022 \mathrm{Re}-0.3534$ \\
& & & If $\mathrm{Re} \geq 25$ & $\mathrm{Nu}=0.0738 \mathrm{Re}+2.9446$ \\
\hline
\end{tabular}

The grid and time step size independency studies are conducted to achieve a convergent and accurate solution in reasonable computational time. Accordingly, three different grid densities of 1.8 million, 3.1 million and 4.3 million cells are tested. With respect to the variation of mean compression space pressure over the cycle, the maximum deviation in this pressure value between the medium and fine meshes does not exceed 2\%. Therefore, the grid of 3.1 million cells is adopted for the current study. In the same manner, three-time step sizes of $7.3 \times 10^{-5} \mathrm{~s}, 5.5 \mathrm{x}$ $10^{-5} \mathrm{~s}$ and $3.06 \times 10^{-5} \mathrm{~s}$ are investigated and, consequently, lead to employ the medium time step size for all the computations presented in this research.

Due to the transient nature of the current case, the convergence of the simulation is based on two main criteria. The first one is the residuals of the transport equations. The convergence criterion for each cycle is set at $1 \times 10^{-6}$ for energy and $1 \times 10^{-3}$ for the rest of the equations. While, monitoring some selected quantities for verifying the attaining of the cyclic steady state solution is the second convergence criterion. The average gas temperature variation of the thirteen subdomains is monitored for this purpose. The deviation in these values should be less than $1 \%$ in two subsequent cycles to reach the cyclic steady state. For each simulation, about 6 cycles are needed for the solution to become cyclic, and each cycle takes about $31.5 \mathrm{~h}$ on a Core i7-6700 acer CPU, $3.4 \mathrm{GHz}$ processor with 16 GB dynamic memory.

\section{Model Validation}

In order to validate the computational results, a comparison of the estimated indicated power from the current simulation with the experimental results of the GPU-3 engine, summarized in Ref. [41], is conducted. Also, the comparison is extended with a range of results of the previous models for the same operating condition, as illustrated in Fig. 4. The default operational condition is listed on the figure.

The comparison indicates that the current CFD simulation has shown a close agreement with the experimental result. By a further insight into the figure, it is noticed that the initial attempts of engine modelling have achieved overestimation in power predictions reached about $80 \%$ or more. However, with coupling the different types of losses, the accuracy of model predictions has noticeably increased upto an 
overestimation of around 10\%. Here, CFD modelling appears to have a higher degree of accuracy, with about $4 \%$ overestimation. The effect of flow leakage from cylinder to buffer space through the piston rings and heat conduction loss through the material thickness, which is not taken into consideration, are partially responsible for the inaccuracy in the current results.

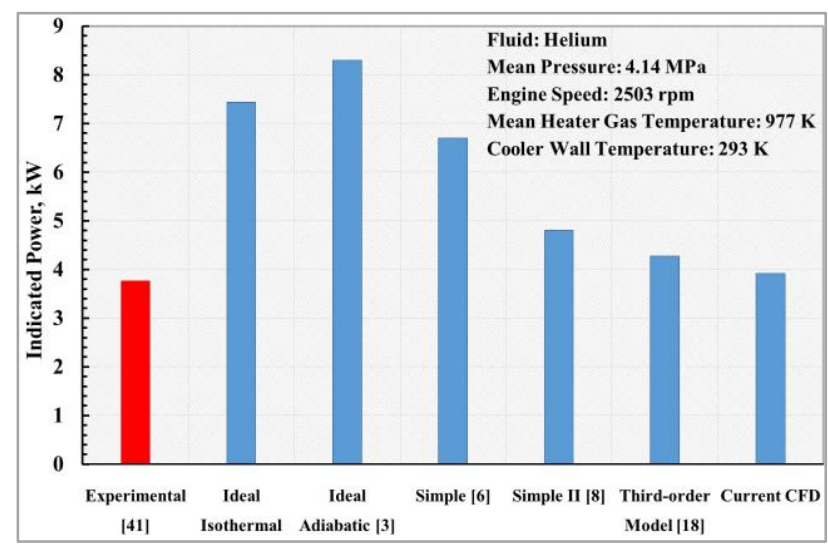

Fig. 4 A comparison of the predicted indicated power between the current CFD simulation, experimental result and previous numerical models.

\section{Results and Discussion}

\subsection{Characterization of the Cyclic Thermal and Fluid Flow Fields}

The instantaneous variations of the total, expansion space, and compression space volumes as functions of the crank angle during a complete cycle are plotted in Fig.5. The total volume includes all the working and dead volume spaces. By comparing Figs. 2 and 5, it can be noticed that the maximum and minimum total volumes of the engine occur at the power piston Bottom Dead Center (BDC) and Top Dead Center (TDC), respectively, resulting in a compression ratio of 1.519 .

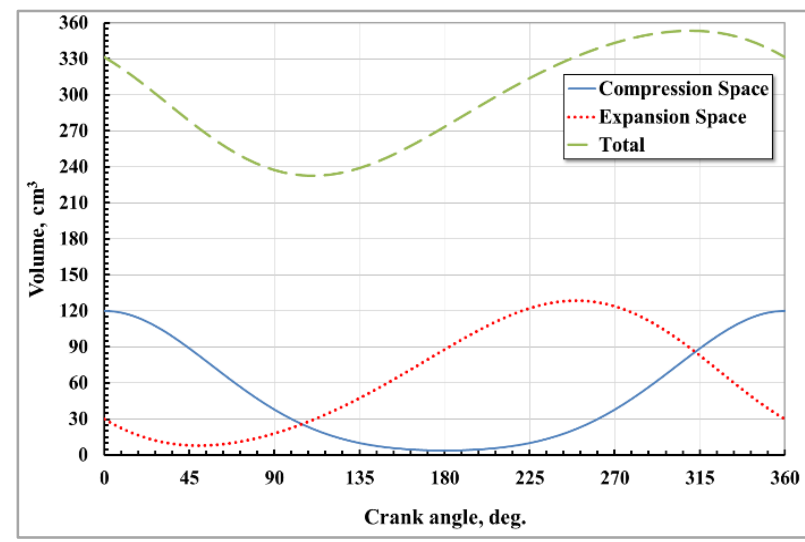

Fig. 5 Instantaneous variations of the total, expansion space, compression space volumes as functions of crank angle $(\theta)$.

Figure 6 shows the instantaneous variations of the average pressure within the expansion space, compression space and on the piston wall as functions of the crank angle over the cycle. The pressure difference between the compression and expansion spaces is the sum of pressure drops in the heater, regenerator, cooler and manifolds with respect to the direction of the flow. Whereas, the difference between the pressure in the compression space and on the piston wall is the pressure loss due to the finite piston speed [8]. In fact, the pressure waves that are generated due to the movement of piston cause the difference between the gas pressure over the moving piston and the bulk gas pressure inside the cylinder according to the flow direction. Therefore, the work delivered to the power piston is usually less than the indicated work.

Here, it should be noted that the error in the power prediction is caused primarily by inaccuracy in the predictions of the pressure phase angle, i.e. the angle between displacer TDC and the maximum gas pressure [42]. For the GPU-3 engine, the maximum gas pressure occurred at approximately $82.5^{\circ}$ after displacer TDC [41] that corresponds to $134^{\circ}$ from the zero-crank angle defined in Fig. 2. In the current simulation, the maximum pressure is attained at $133.2^{\circ}$.

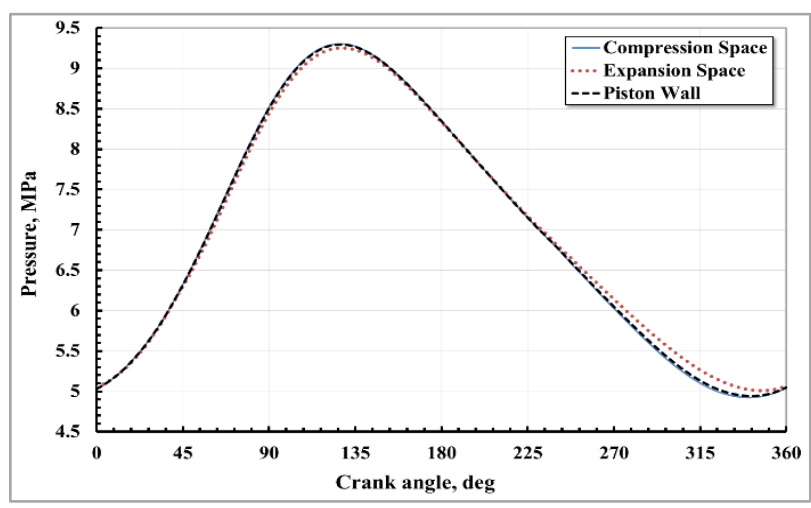

Fig. 6 Instantaneous variations of the average pressure in compression; expansion spaces and on the piston wall as functions of crank angle $(\theta)$.

The instantaneous variations of average gas temperature within the compression space, expansion space, heater, cooler and regenerator versus the crank angle during the cycle are represented in Fig. 7.

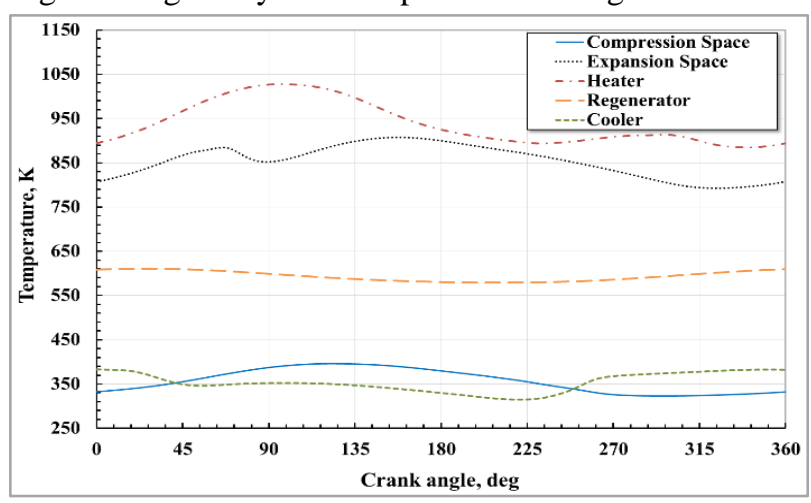

Fig. 7 Instantaneous variations of the average gas temperature within the compression space, expansion 
space, heater, cooler and regenerator as functions of crank angle $(\theta)$.

Apparently, the temperature variation in each volume is far from the harmonic distribution. This result contradicts with those predicted from the second- and third-order models for the GPU-3 engine [4, 6, 8, 16]. which confirmed that the temperature distribution was significantly near the sinusoidal profile. This is attributed to the complex interaction between the fluid dynamics and heat transfer phenomena that occur within the engine and cannot be captured by these models. Therefore, the detailed CFD results should be represented to demonstrate these phenomena and their effects on the thermal and fluid flow fields inside the engine. Accordingly, the temperature contours of the entire flow domain for eight different crank angles during a complete cycle are illustrated in Fig. 8.

At the beginning of the cycle $\left(\theta=0^{\circ}\right)$, when the volume of the compression space is at maximum, the power piston and displacer is near their BDC and TDC, respectively, as shown in Fig. 2. They move upwards causing a simultaneous compression for both the compression and expansion spaces. At this instant, the gas moves from the expansion space toward the compression one through two paths, the appendix gap and the three-heat exchangers. As can be observed from Fig. 8 (a), the mixing between the hot gas jet from the appendix gap and the less hot one from the coldend connection occurred within the compression space causes the appearance of a relatively hot thermal plume in the space.

With the continuous movement of the displacer towards its TDC, which occurred at $51.4^{\circ}$, the velocity of the displacer decreases, leading to a gradual reversal of the flow direction, until it fully turns from the compression to the expansion space. At $\theta=45^{\circ}$, the displacer is close to its TDC and the gas is flowing from the compression to the expansion space through the two paths. However, a small amount of hot gas still exits from the expansion space through the heater pipes, which is partially closed by the displacer. At this exact moment, the direction of flow is reversed through the heater pipes. After reaching to TDC and moving downwards, the displacer allows the expansion space to be expanded. When the displacer is accelerated downwardly, the amount of the cold flow coming from the compression space through the appendix gap into the expansion space is also increased. This phenomenon explains the sudden reduction in the average gas temperature of the expansion space at the beginning of its expansion stroke, as clarified in Fig. 8 at crank angle of about $90^{\circ}$. When the piston reaches its TDC $\left(\theta=110^{\circ}\right)$ and starts to reverse its direction, the compression space is fully compressed. Hence, large amounts of flow are ejected from this space in the direction to expansion space. As it can be seen in Fig. 7 , the period from $\theta=100^{\circ}$ to $165^{\circ}$ is characterized by an obvious increase in the temperature of the expansion space. This also explains the higher gas temperatures that are observed in heater during this period. Consequently, the cycle pressure continues to rise in spite of the increase in the engine total volume, Fig.6. During the expansion stroke of the expansion space, the dominant feature of the flow field is the appearance of a pair of counter-rotate vortices with different strengths. This flow feature is more pronounced during the period of $180^{\circ}$ to $225^{\circ}$, Fig. 8 (e-f), as the piston velocity is maximum. Although, these vortices enhance the mixing within the space, the average gas temperature of this space decreases markedly due to continuous of the expansion stroke, as depicted in Fig.8. 


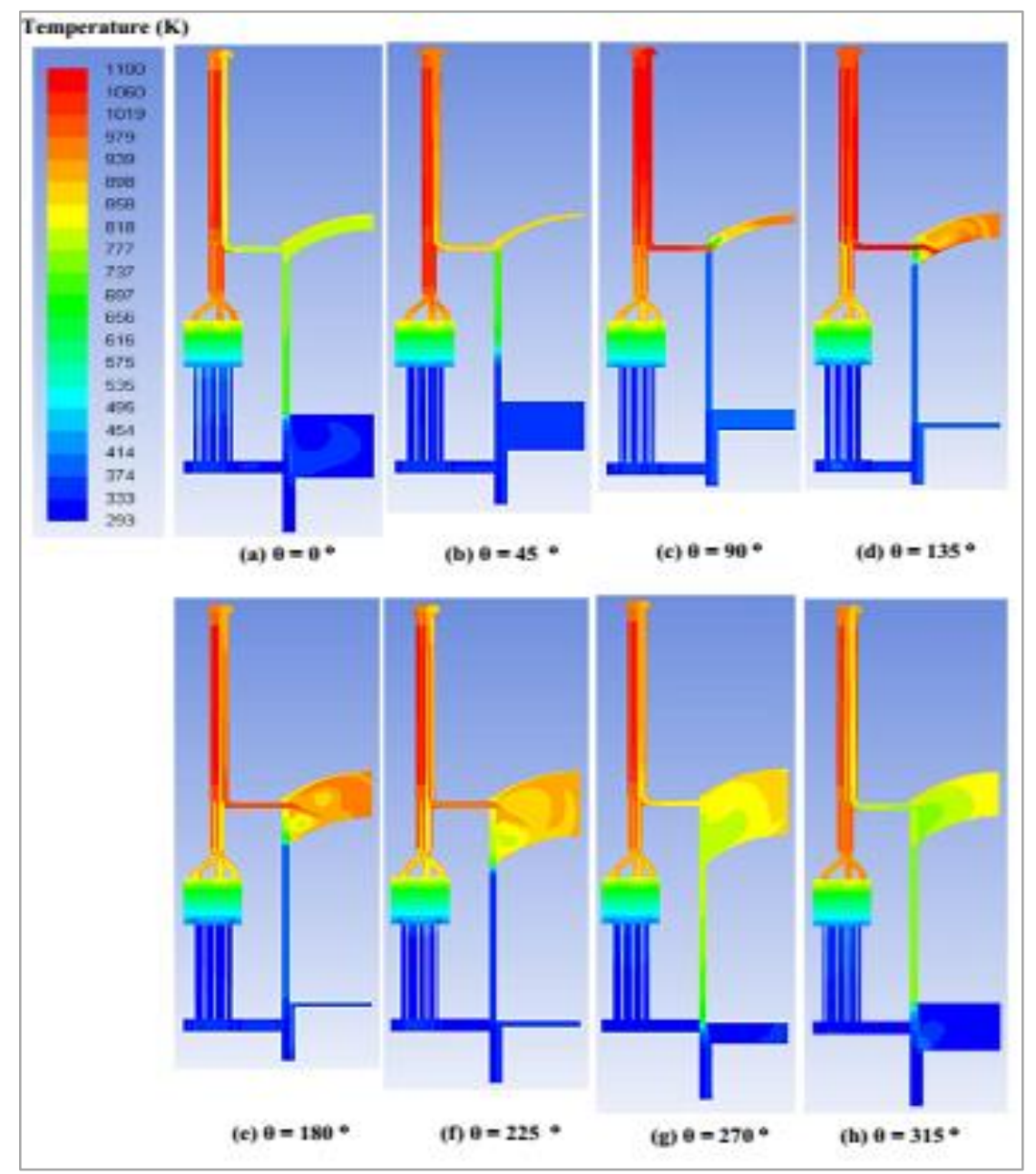

Fig. 8 A 2D view of gas temperature contours through the engine at eight different crank angles.

As the displacer becomes near its BDC, the flow reverses its direction incrementally for the second time, to turn from the expansion to the compression space. Figure $8(\mathrm{~g}-\mathrm{h}, \mathrm{a})$ illustrate the induction of the gas into the compression space during its expansion stroke through the appendix gap and the heat exchangers. It can be noticed from Fig. 7 that a nearly constant gas temperature, throughout the compression space, is noticed during this period. This occurs due to two contradicting effects, the heating by the two gas jets incoming from the hot space and the cooling accompanying the volume expansion.

\subsection{Energy Analysis of Stirling Engine}

The $\mathrm{p}-\mathrm{V}$ diagram of the compression and expansion spaces is shown in Fig. 9. From this figure, the indicated power, $\mathrm{P}_{\text {ind }}$, of the GPU-3 engine can be predicted by integrating the enclosed area of the two diagrams as follows:

$$
P_{\text {ind }}=\left(\frac{\omega_{r}}{2 \pi}\right)\left[\oint p_{e} d V_{e}+\oint p_{c} d V_{c}\right]
$$

Using the data displayed in Fig. 6, the power delivered to the power piston surface, $\mathrm{P}_{\mathrm{p}}$, can be also determined, by the following equation:

$$
\mathrm{P}_{\mathrm{p}}=\left(\frac{\omega_{\mathrm{r}}}{2 \pi}\right) \oint\left(\int_{\text {piston }} \mathrm{pdA}\right) \mathrm{dy}
$$

Where $\mathrm{A}$ is the area of the piston top surface. The difference between the indicated power and the piston power represents the piston finite speed power loss.

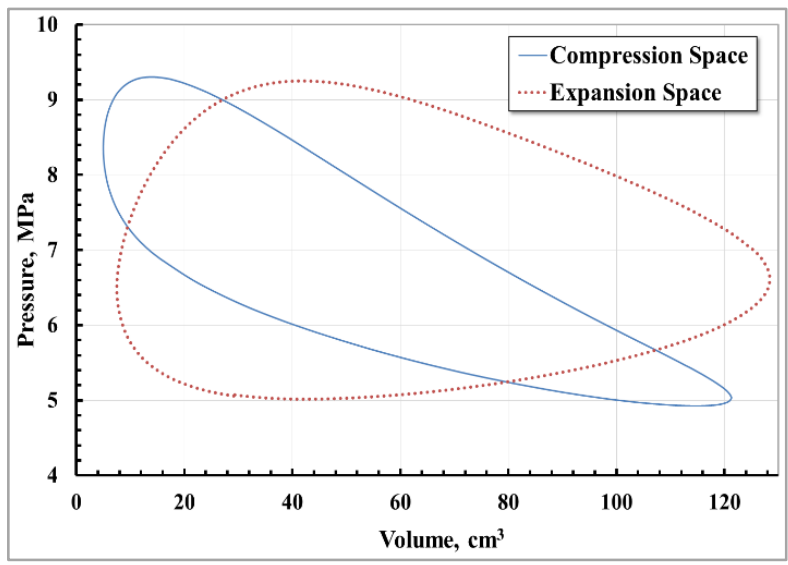

Fig. $9 \mathrm{p}-\mathrm{V}$ diagrams of the compression and expansion spaces.

Figure 10 illustrates the instantaneous variations of both the added heat rate to the heater and the rejected heat rate out of the cooler. From this figure, the cyclic rate of heat addition in the heater as well as the heat 
rejection out of the cooler can be computed from the integration:

$$
\mathrm{Q}=\left(\frac{\omega_{\mathrm{r}}}{2 \pi}\right) \oint\left(\int_{\text {wall }} \mathrm{q}^{\cdot} \mathrm{dA}\right) \mathrm{dt}
$$

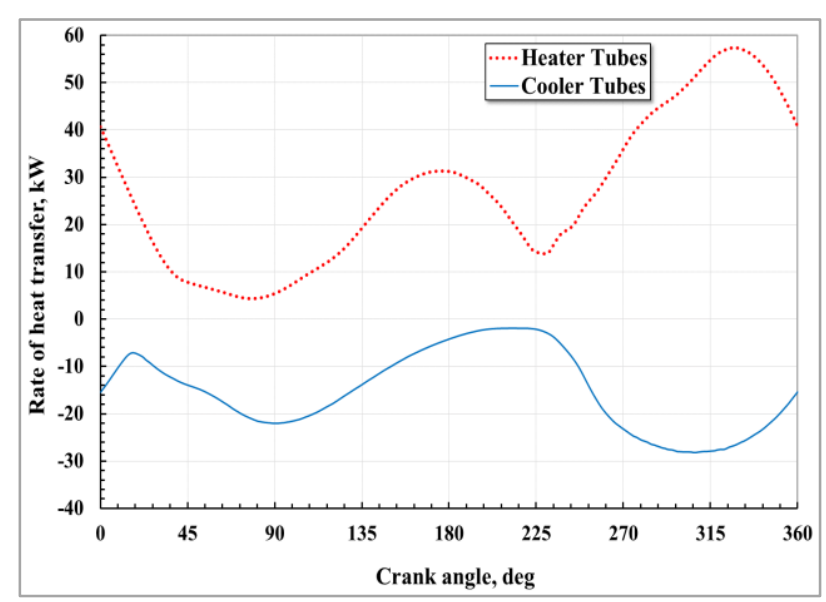

Fig. 10 Instantaneous variations of the added heat rate to the heater and the rejected heat rate out of the cooler as functions of crank angle $(\theta)$.

It should be pointed out that the losses mechanisms mainly depend on the unsteady behaviors of both thermodynamics, fluid dynamics and consequently, heat transfer phenomena within the engine. Hence, the losses-related phenomena along with the method of calculating each loss are addressed in the following subsections.

\subsubsection{Hysteresis Loss}

The cyclic heat transfer rate, heat pumping, through the walls of the working spaces is usually referred to as "hysteresis loss". This occurs due to the heat interaction between the working gas and the walls of these spaces. Tew [21] pointed out that the other regions with small surface-to-volume ratio, such as manifolds, are also responsible for the hysteresis loss.

\subsubsection{Working Spaces}

Figure 11 shows the effect of the fluid flow field on the local directive heat transfer coefficient on the walls of the expansion space at $\theta=180^{\circ}$. Here, the directive heat transfer coefficient has the same value of the traditional heat transfer coefficient but with a sign for identifying the direction. The positive sign corresponds to heat transfer from the walls to the fluid region, while, negative sign correspond to heat transfer in the opposite direction. Interestingly, it can be observed that there is a wide range of variations of this coefficient on each wall. This observation confirms and agrees with that of Chen et al. [30] about the weakness of the assumption of a constant heat transfer coefficient.

As pointed out previously, the flow jetting from the appendix gap and heater within the expansion space is generally accompanied by the appearance of two counter-rotate vortices, so-called tumbles. Tumble vortex is a well-known term in reciprocating engine, indicating a rotational motion about a cylinder circumferential axis. This 3D phenomenon and its effects appear clearly in Fig. 11. The dominant tumble vortex that is driven by the hot jet coming in from the heater pipes heats up the top wall of the cylinder due to the impingement heat transfer. Meanwhile, the side wall of the cylinder is exposed to two simultaneous different effects. It is rapidly cooled down by the cold flow of the corner tumble vortex that is formed by the jetting from the appendix gap. This effects gradually decreases upwards due to the superiority of the heating effect by the hot dominant vortex. The consequence of these contradicting effects is that the heat transfer coefficient changes from high positive values at the impinging region of the corner vortex to relatively high negative values at the impinging region of the dominant vortex.

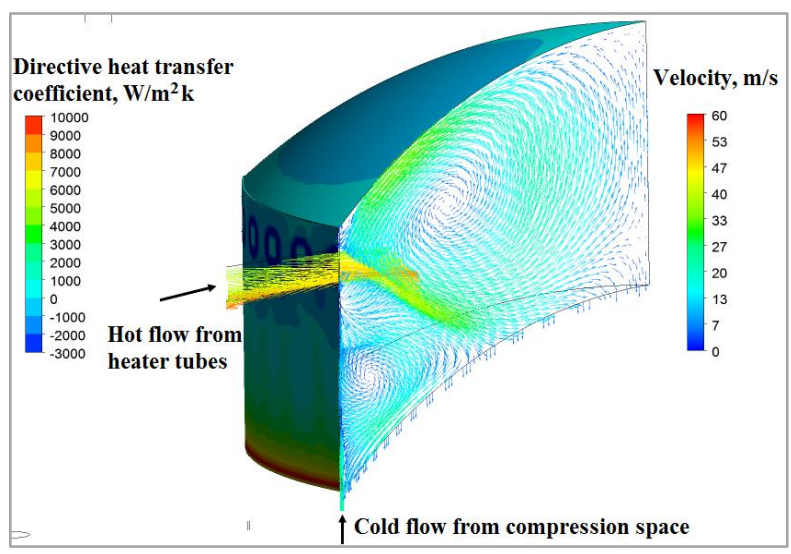

Fig. 11 Effect of the fluid flow field on the local directive heat transfer coefficient on the walls of the expansion space at $\theta=180^{\circ}$.

The instantaneous variation of the convective heat transfer rate in the expansion space, as a function of the crank angle, can be displayed in Fig. 12. During early part of the cycle, the trends of the heat transfer rates at the top and side walls of the expansion space are nearly similar. However, after the jetting of flow incoming from the compression space, from about $51.4^{\circ}-250^{\circ}$ of crank angle, obvious differences start to appear. This is attributed to the different effect of the tumble vortex on each wall, as discussed above. During the compression stroke of the expansion space, which is accompanied by the ejection of the flow from it, a markedly decrease in the rates of heat transfer is noticed. It can be attributed to the disappearance of the tumble vortices and its impinging effects. This result is inconsistent with that reported by Chen et al. [30], who emphasized that the ejection process promotes the heat transfer in a way similar to the impingement does.

In the same manner, the effect of the fluid flow field on the convective heat transfer processes within the compression space can be explained. Figure 13 also 
represents this effect in terms of the directive heat transfer coefficient. A similar impingement phenomenon can be observed in the compression space, however, the two jets coming from the expansion space forms a single tumble vortex. This vortex causes a rapid heating for the side wall of the space. Another indirect effect of these jets appears on the piston. It may be due to the jet entrainment that draws the bulk flow within the space in its direction inducing a secondary vortex rotating in reverse direction. This vortex cools up the bottom of the side wall by bringing colder fluid to be in vicinity of it. Furthermore, it has a decreasing rate of heat transfer from the piston wall by inducing the cold flow away from it.

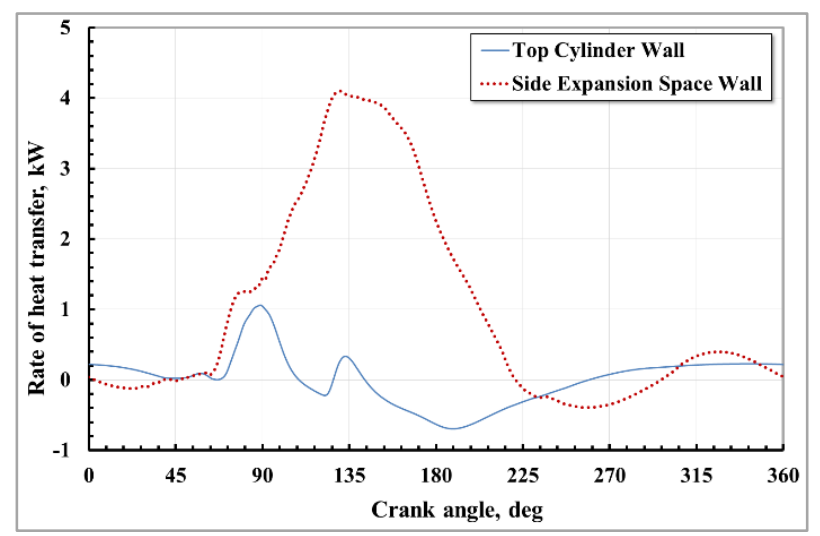

Fig. 12 Instantaneous variations of the convective heat transfer rates in the expansion space as a function of crank angle $(\theta)$.

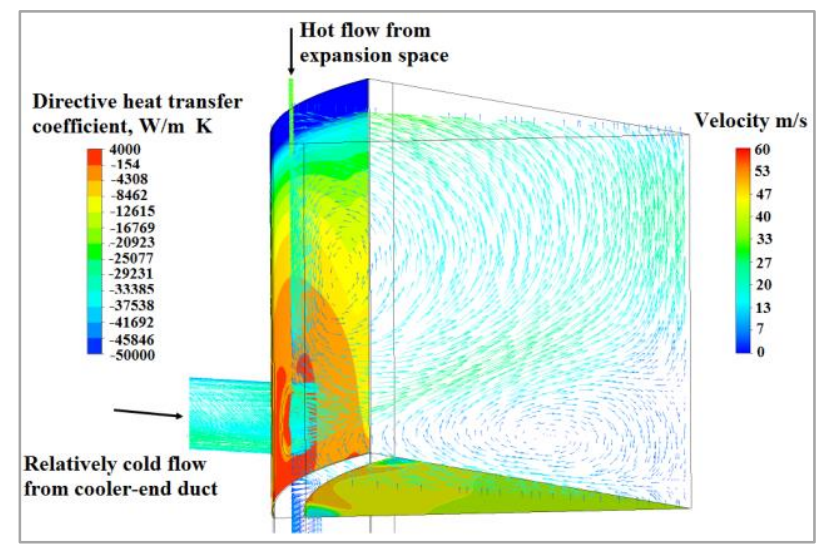

Fig. 13 Effect of the fluid Flow field on the local directive heat transfer coefficient at the walls of the compression space at $\theta=0^{\circ}$.

The instantaneous variation of the convective heat transfer rate at the side wall of compression space can be presented, Fig 14. However, it is completely different for the piston, due to its motion without being directly exposed to impingement heat transfer. With the upward motion of the piston, it becomes exposed to a relatively hot flow of gas within the compression space which heats it up. After the reversal of its direction, the gas cools down causing the heat flowing in the reversed direction. There are some distortions in the variation of piston heat transfer rate which occur when the piston reverses its direction.

Here, it is concluded that, within the compression and expansion spaces, the dominant heat transfer rates occur during the expansion strokes. This is due to the significant impinging effect of the tumble vortices generating from the flow jetting within the spaces .

From Figs. 12 and 14, the cyclic convective heat transfer rate from the walls of the compression and expansion spaces can be calculated according to eq. 28 . These values represent the hysteresis losses from the working spaces.

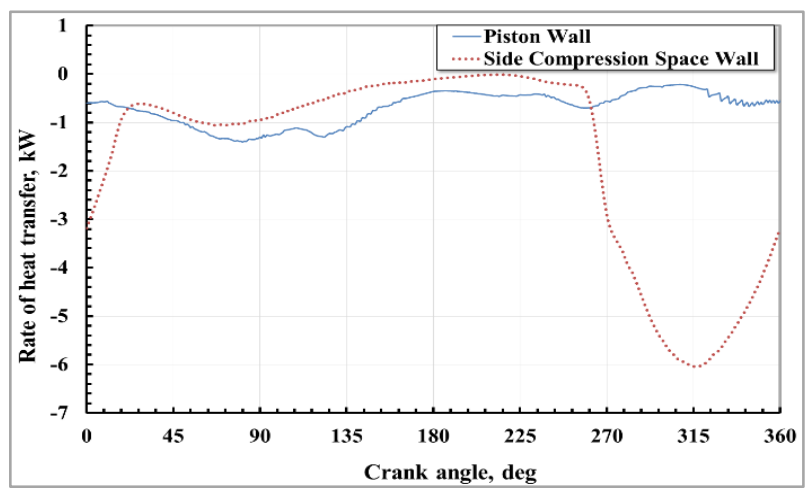

Fig. 14 Instantaneous variations of the convective heat transfer rates in the compression space as a function of crank angle $(\theta)$.

\subsubsection{Manifolds of Hot and Cold Spaces}

Generally, the manifolds of the Stirling engine are divided into the manifolds of the hot space and manifolds of the cold space. In the GPU-3 engine, the heater header and the parts of the heater tubes that are not exposed to the heat source are considered the hot space manifolds. Whereas, the cooler - end connection as well as the parts of the cooler tubes that are not exposed to the cooling water are the manifolds of the cold space. Figure 15 represents the instantaneous variation of the convective heat transfer rate in the engine manifolds as a function of the crank angle. From this figure, the hysteresis loss from engine manifolds can be calculated according to eq. 28 .

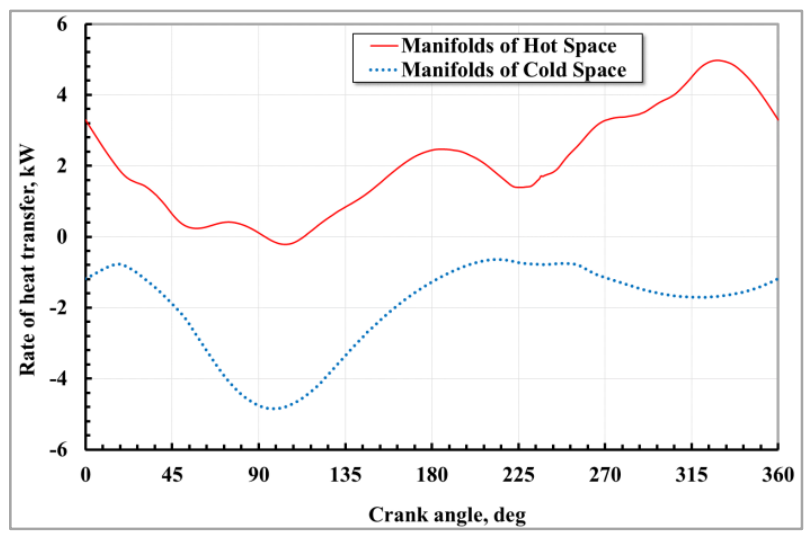

Fig. 15 Instantaneous variations of the convective heat transfer rates in the manifolds of hot and cold spaces as a function of crank angle $(\theta)$. 


\subsubsection{Thermal Loss of Regenerator}

Figure 16 illustrates the flowing of gas through the regenerator that involves two periods, namely, hot and cold blow. During the hot blow period, the regenerator matrix acts as a thermal storage element that absorbs energy from the hot gas flowing from the heater. Then, it gives up this energy to the cold gas flowing from the cooler during the cold blow period. Accordingly, the heat transfer coefficient between the gas and solid matrix, and subsequently, the temperature difference between them changes continuously over time. The instantaneous variations of the flow Reynolds number and the heat transfer coefficient between matrix and gas within the regenerator versus the crank angle are illustrated in Fig. 17.

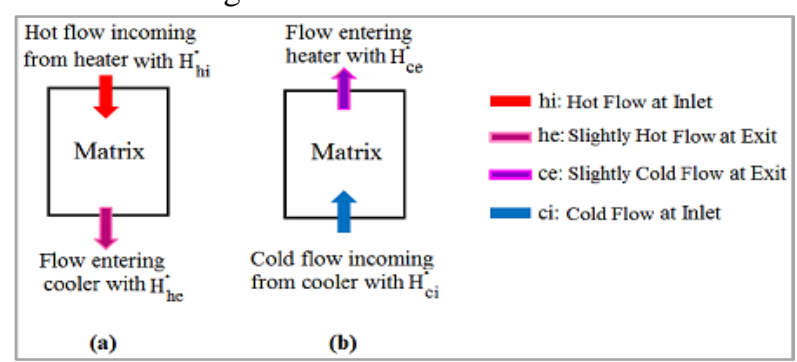

Fig. 16 Schematic representation of heat exchange between the gas and matrix within regenerator during:

(a) hot blow period and (b) cold blow period.

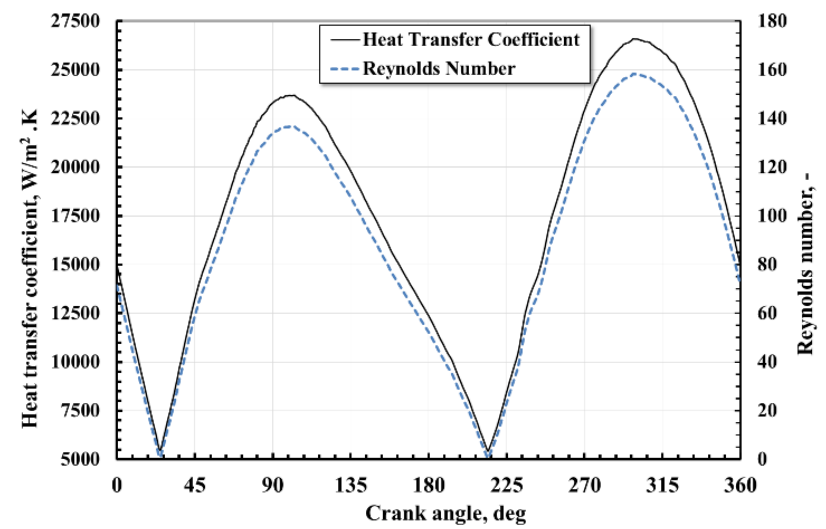

Fig. 17 Instantaneous variations of the heat transfer coefficient and flow Reynolds number within regenerator as functions of crank angle $(\theta)$.

Figure 18 presents the temperature contours for the fluid and solid matrix through the regenerator, during the cold blow period at $\theta=135^{\circ}$. From this Figure, it is indicated that there are slightly higher temperatures for the solid matrix than the gas. These are clearly observed due to the effective heat interaction between the matrix and the gas at this moment. Furthermore, the jetting regions into the matrix is clearly identified by a high axial temperature gradient, as illustrated in the figure. This is due to the high rate of heat transfer that occurs at the inlet and progressively decreases along its travel in the regenerator. Another phenomenon that accompanies the flow jetting into the regenerator, denoted as the inflow induced matrix temperature oscillation (IIMTO), appears. This phenomenon was previously discussed by Andersen et al. [43].

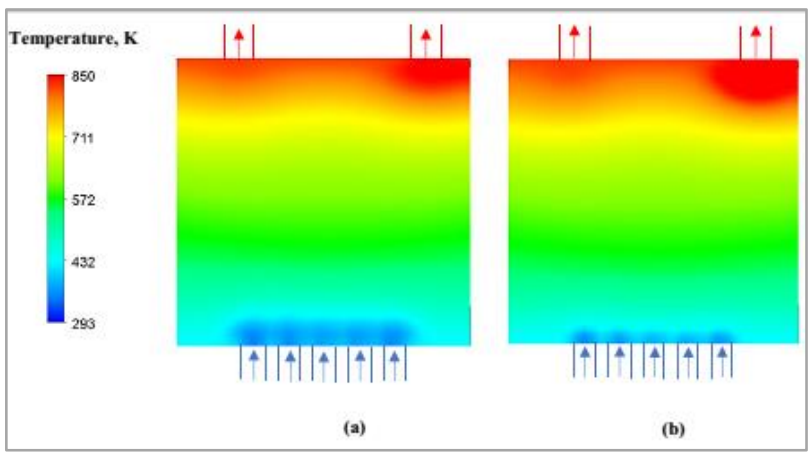

Fig. 18 Temperature contours of the (a) fluid and (b) solid matrix through the regenerator, during the cold blow period at $\theta=135^{\circ}$.

Figure 18 also illustrates the heat exchange, based on enthalpy flow rate, between the gas and matrix within regenerator during the hot and cold blow periods. Here, $\mathrm{H}^{\cdot}$ denotes the instantaneous enthalpy flow rate of the fluid. The instantaneous enthalpy flow rate of the fluid at a specific cross - sectional area is determined by:

$$
\mathrm{H}^{\cdot}=\int_{\text {area }} \rho \mathrm{u} \mathrm{C}_{\mathrm{p}}(\mathrm{T})\left(\mathrm{T}-\mathrm{T}_{\text {ref }}\right) \mathrm{dA}
$$

where $\mathrm{Cp}$ and $\mathrm{A}$ are the specific heat and the crosssectional area, respectively. $\mathrm{T}_{\text {ref }}$ is the reference temperature for the enthalpy calculation.

Figure 19 shows the instantaneous enthalpy flow rate of the fluid at both ends of the regenerator, at heater / regenerator and cooler / regenerator interfaces, along with the net rate between both ends. Here, the positive values indicate the heat transfer from the regenerator matrix to the fluid and vice versa.

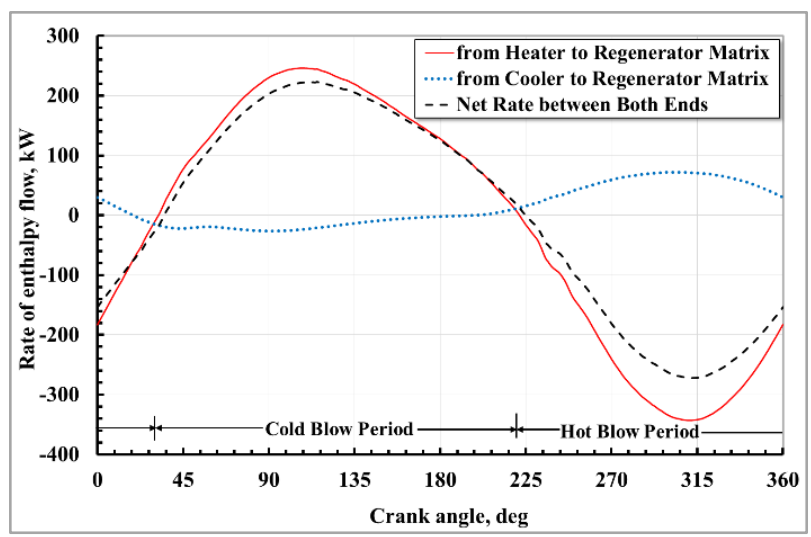

Fig. 19 Instantaneous variations of the enthalpy flow rate of fluid at both sides of regenerator along with net rate between the both ends as functions of crank angle $(\theta)$.

As expected, no complete heat recovery occurred within the regenerator. This is attributed to several losses taking place within regenerator. These are the axial conduction loss through the matrix and the gas, convective heat loss to the regenerator walls and swing losses. Besides, the inability of the matrix to store all the available energy in the hot fluid or completely 
release it to the cold gas due to its effectiveness., the thermal heat loss through the regenerator, $Q_{\text {loss,r }}$, can be calculated by the cyclic integration of the instantaneous net enthalpy flow rate, $\mathrm{H}_{\text {net }}$, between the two ends of the regenerator as:

$$
\mathrm{Q}_{\mathrm{loss}, \mathrm{r}}=\frac{\omega_{\mathrm{r}}}{2 \pi} \oint \mathrm{H}_{\text {net }}^{\cdot} \mathrm{dt}
$$

Finally, it should be emphasized that the incomplete heat recovery in the regenerator requires additional heat to be supplied in the heater and more heat to be rejected in the cooler to compensate the non-ideal operation of the regenerator. This, consequently, leads to a noticeable reduction in the engine performance.

\subsubsection{Displacer and Appendix Gap}

In fact, the fluid flow within the appendix gap as well as the flow of heat through the displacer are considered of the most complex phenomena occurring in Stirling engine. With respect to the appendix gap, the fluid flow through it has been previously addressed in section 6.1. However, the effect of this fluid dynamics on the enthalpy pumping loss still needs analysis. The instantaneous enthalpy flow rate at the inlet of the appendix gap, $\mathrm{H}_{\mathrm{g}}$, can be calculated as:

$$
H_{g}=2 \pi \int_{R_{d}}^{R_{d}+J}\left(\rho u C_{p}(T)\left(T-T_{r e f}\right)\right) r d r
$$

where $R_{d}$ and $J$ are the radius of the displacer and the width of the appendix gap, respectively. Accordingly, Fig. 20 shows the instantaneous enthalpy flow rate of the fluid entering the appendix gap from the expansion space. The figure also displays the pressure difference between the expansion and compression spaces.

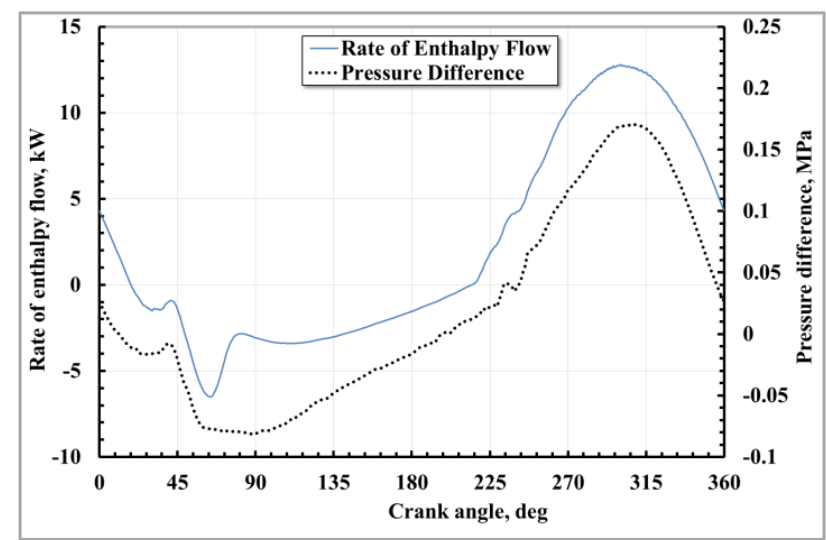

Fig. 20 Instantaneous variations of enthalpy flow rate of fluid entering the appendix gap from expansion space and pressure difference between expansion and compression spaces as functions of crank angle $(\theta)$.

From this figure, it is noticeable that the pressure difference between the two working spaces is the main driver for the mass flow through the appendix gap. This appears from the matching of the two trends except in period from $63.9^{\circ}$ to $109.8^{\circ}$. As, the displacer direction is reversed, and consequently, allowing for a gradual opening of the heater tubes to pour the fluid within the expansion space. This partially obstruct the flow induced through the appendix gap, as shown in Fig. 8 (c). The matching between the pressure difference and the enthalpy flow confirms the prevailing of the pressure difference effect over the other effects: temperature gradient, displacer motion and heat exchange between the appendix gap and displacer walls.

The enthalpy pumping loss, $\mathrm{Q}_{\text {loss,g }}$, can be calculated by the cyclic integration of the instantaneous enthalpy flow rate at the inlet of the appendix gap, $\mathrm{H}_{\mathrm{g}}$, as:

$$
\mathrm{Q}_{\mathrm{loss}, \mathrm{g}}=\frac{\omega_{\mathrm{r}}}{2 \pi} \oint \mathrm{H}_{\mathrm{g}}^{\cdot} \mathrm{dt}
$$

Regarding the displacer, the heat is axially conducted through it under the effect of the temperature gradient between the compression and expansion spaces. Besides, the reciprocating motion of the displacer shuttles extra heat transfer between the two spaces, shuttle heat loss. The jetting of the hot fluid over the displacer top wall significantly enhances the rates of shuttle heat loss, as shown in Fig. 21. Also, the exposure of the bottom wall of the displacer to rapid cooling due to the expansion of the compression space has the same effect.

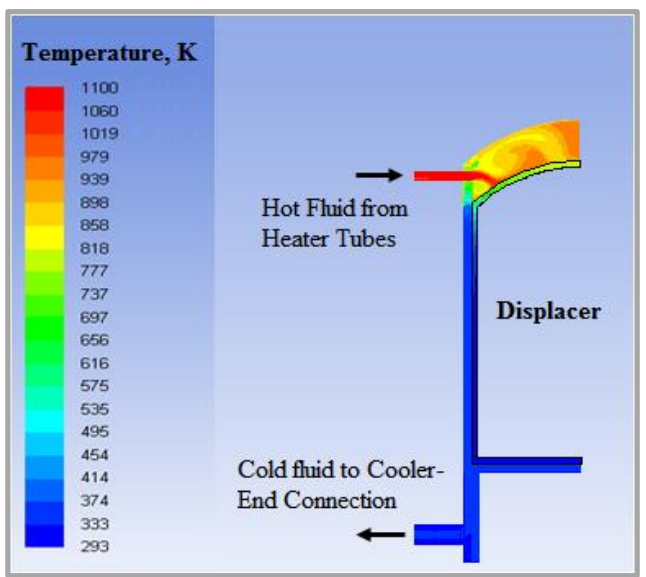

Fig. 21 Jetting of the hot fluid over the displacer top wall at $\theta=150^{\circ}$.

The instantaneous rate of heat conduction at the displacer top wall can be determined through the equation:

$$
\mathrm{Q}_{\text {cond,d }}=\int_{\text {area }}-\left.\mathrm{K}_{\mathrm{f}} \frac{\partial \mathrm{T}_{\mathrm{f}}}{\partial \mathrm{n}}\right|_{\mathrm{DTW}} \mathrm{dA}
$$

where $\mathrm{K}_{\mathrm{f}}$ and $\mathrm{T}_{\mathrm{f}}$ are the thermal conductivity and the local temperature of the fluid and the top wall of the displacer. DTW refers to displacer top wall. Figure 22 illustrates the instantaneous variation of the rate of heat conduction at the displacer top wall. From this figure, the shuttle heat loss, $Q_{\text {shuttle, can be calculated }}$ according to the following equation: 


$$
\mathrm{Q}_{\text {shuttle }}=\frac{\omega_{\mathrm{r}}}{2 \pi} \oint \mathrm{Q}_{\text {cond,d }} \mathrm{dt}
$$

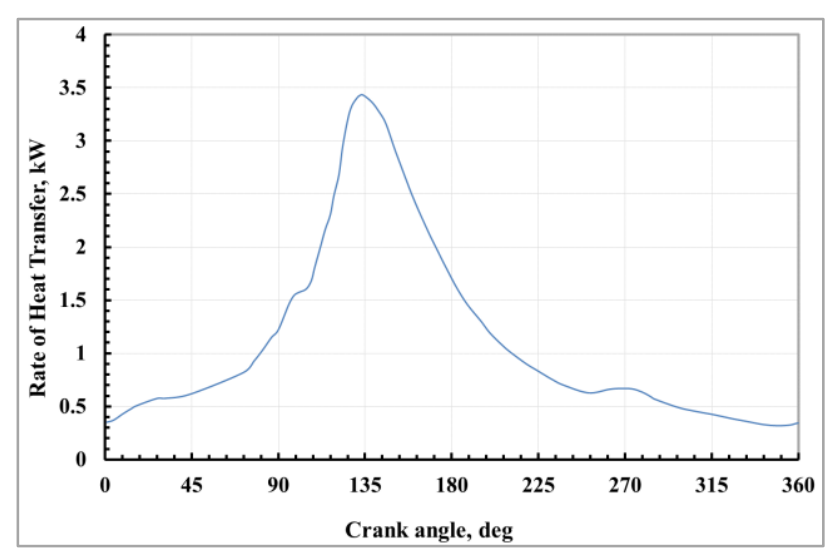

Fig. 22 Instantaneous variations of the conductive heat transfer rates at the top wall of the displacer as a function of crank angle $(\theta)$.

\subsubsection{Pumping Loss}

In order to calculate the pumping loss, the pressure drop in each component of the engine should be estimated. Figure 6.20 presents the instantaneous variation of the pressure drop through the engine components; regenerator, heater and its header as well as cooler and its end - connection. The pressure drop in the entrance and the exit of each component is included. As expected, the pressure drop in the regenerator represents the main contributor on the total pressure drop within the engine. Meanwhile, it is observed that the pressure drops in the heater and its header as well as the cooler and its end connection are also significant values. This result conflicts with the results of Babaelahi and Sayyaadi [8] and Tlili et al. [44]. Accordingly, the pumping loss can be calculated by:

$$
\mathrm{P}_{\text {pumping }}=\frac{|\Delta \mathrm{p}| \mathrm{m}}{\rho_{\mathrm{m}}}
$$

where $\Delta \mathrm{p}, \mathrm{m}$ and $\rho_{\mathrm{m}}$ are the pressure drop, the mass flow rate and the mean value of the density through each component, respectively.

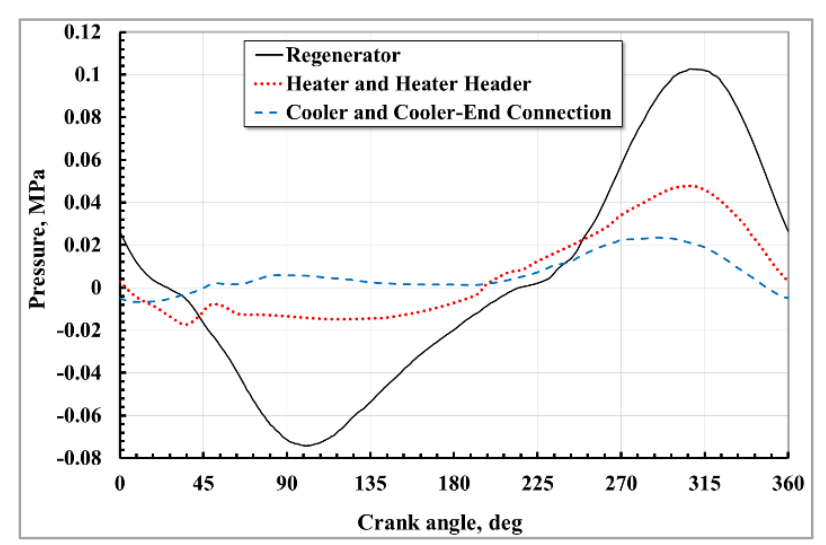

Fig. 23 Instantaneous variations of pressure drop through regenerator, heater and heater header as well as cooler and cooler - end connection.

As presented in the previous subsections, the hysteresis loss, the regenerator heat loss, the enthalpy pumping and the shuttle losses can be calculated. An energy balance representation for the GPU-3 Stirling engine is illustrated in Fig. 24.

According to this energy balance, the pumping power and the regenerator thermal losses represent the largest part of the Stirling engine losses. The thermal efficiency of the GPU-3 Stirling engine, based on the power piston, is of about $36.6 \%$.

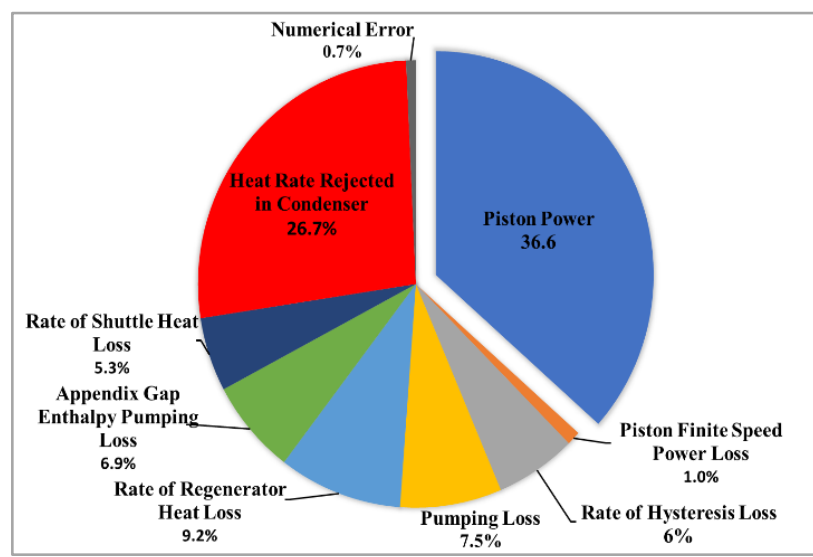

Fig. 24 Energy balance of the GPU-3 Stirling engine.

\section{Conclusion}

A three-dimensional Computational Fluid Dynamics (CFD) simulation for the GPU-3 Stirling engine is performed. Firstly, a thorough characterization of the thermal and fluid flow fields during the cycle is presented. Furthermore, the effects of the unsteady behaviors of both thermodynamics and fluid dynamics on the heat transfer phenomena within the engine components are also investigated. Finally, a comprehensive energy analysis for the engine is conducted to accurately identify the sources and magnitudes of thermodynamic losses. The computational results show that:

- A close agreement with the experimental results with an accuracy of about $96 \%$.

- The instantaneous variation of the average gas temperature within each component of the engine is far from the harmonic distribution due to the complex interaction between the fluid dynamics and the heat transfer phenomena that occurs within the engine

- Within the compression and expansion spaces, the dominant heat transfer rates occur during the expansion strokes due to the significant impinging effect of the tumble vortices generating from the flow jetting .

- The jetting and ejecting processes into the regenerator are characterized by a significant 
temperature gradient and a large matrix temperature oscillation .

- The pressure difference between the expansion and compression spaces is the main driver for the flow leakage through the appendix gap.

- From the energy analysis, the regenerator thermal loss and the pumping power represent the largest part of the Stirling engine losses by about $9.2 \%$ and $7.5 \%$, respectively.

\section{References}

[1] U. E. I. A. (EIA), "Renewable Energy Explained," 2018. [Online]. Available: https://www.eia.gov/energyexplained.

[2] B. Kongtragool and S. Wongwises, "A Review of Solar-Powered Stirling Engines and Low Temperature Differential Stirling Engine," Renewable Sustainable Energy Rev., vol. 7, p. 131-154, 2003.

[3] W. Martini, "Stirling Engine Design Manual," DOE/NASA/3194-1, NASA CR-168088, 1983.

[4] M. Babaelahia and H. Sayyaadi, "Modified PSVL: A Second Order Model for Thermal Simulation of Stirling Engines Based on Convective-Polytropic Heat Transfer of Working Spaces," Applied Thermal Engineering, vol. 85, pp. 340-355, 2015.

[5] T. Finkelstein, "Generalized thermodynamic analysis of Stirling engines," Society of Automotive Engineer (SAE), no. 118B, 1960.

[6] I. Urieli and D. Berchowitz, Stirling cycle engine analysis, Adam Hilger Ltd, 1984.

[7] Y. Timoumi, I. Tlili and S. Nasrallah, "Design and Performance Optimization of GPU-3 Stirling Engines," Energy, vol. 33, p. 1100-1114, 2008.

[8] M. Babaelahia and H. Sayyaadi, "Simple-II: A New Numerical Thermal Model for Predicting Thermal Performance of Stirling Engines," Energy, vol. 69, pp. 873-890, 2014.

[9] S. Kaushik and S. Kumar, "Finite Time Thermodynamic Analysis of Endoreversible Stirling Heat Engine with Regenerative Losses," Energy, vol. 25, p. 989-1003, 2000.

[10] M. H. Ahmadi, M. A. Ahmadi, F. Pourfayaz, M. Bidi, H. Hosseinzade and M. Feidt, "Optimization of Powered Stirling Heat Engine with Finite Speed Thermodynamics," Energy Conversion and Management, vol. 108, p. 96-105, 2016.

[11] H. Hosseinzade and H. Sayyaadi, "CAFS: The Combined Adiabatic-Finite Speed Thermal Model for Simulation and Optimization of Stirling Engines," Energy Conversion and Management, vol. 91, p. 32-53, 2015.

[12] G. Barreto and P. Canhoto, "Modelling of a Stirling Engine with Parabolic Dish for Thermal to Electric Conversion of Solar Energy," Energy Conversion and Management, vol. 132, p. 119 -
$135,2017$.

[13] S. Andersen, Numerical Simulation of Cyclic Thermodynamic Processes, Ph.D. Dissertation, Technical University of Denmark, Lyngby, Denmark, 2006.

[14] T. Finkelstein, "Thermodynamic analysis of Stirling engines," J. of Spacecraft Rockets, vol. 4, p. 1184-1189, 1967.

[15] I. Urieli, C. Rallis and D. Berchowitz, "Computer simulation of Stirling cycle machines," in In: Proc 12th IECEC, Paper No. 779252, 1977.

[16] R. Tew, "Computer Program for Stirling Engine Performance Calculations," NASA TM-82960, DOE/NASA/51040-42, 1983.

[17] J. Escalona, D. Sánchez, R. Chacartegui and T. Sánchez, "Model of Performance of Stirling Engines," in Proceedings of ASME Turbo Expo 2012, Copenhagen, Denmark, GT2012-69729, 2012.

[18] S. Toghyani, A. Kasaeian, S. Hashemabadi and M. Salimi, "Multi-Objective Optimization of GPU3 Stirling Engine Using Third Order Analysis," Energy Conversion and Management, vol. 87, p. 521-529, 2014.

[19] I. Barreno, S. Costa, M. Cordon, M. Tutar, I. Urrutibeascoa, X. Gomez and G. Castillo, "Numerical Correlation for the Pressure Drop in Stirling Engine Heat Exchangers," Int. J. of Thermal Sciences, vol. 97, pp. 68-81, 2015.

[20] J. Salazar and W. Chen, "A Computational Fluid Dynamics Study on the Heat Transfer Characteristics of the Working Cycle of a $\beta$-Type Stirling Engine," Energy Conversion and Management, vol. 88, pp. 177-188, 2014.

[21] R. Tew, "Overview of Heat Transfer and Fluid Flow Problem Areas Encountered in Stirling Engine Modeling," NASA TM-100131, 1988.

[22] R. Tew and M. Ibrahim, "Two-Dimensional Compressible Non-Acoustic Modeling of Stirling Machine-Type Components," J. of Propulsion and Power, vol. 19, no. 5, pp. 922-929, 2003.

[23] D. Gedeon, "A Computer Program for Modeling Two-Dimensional Gas Flow in Stirling Engine Regenerators," Final Report. Prepared for NASA Lewis Research Center under Contract DEN3308, Gedeon Associates, 1986.

[24] M. Ibrahim, M. Mittal, T. Simon and D. Gedeon, "A 2-D CFD Model of a Free Piston Stirling Engine for Space Applications with Annular Heat Exchangers," in 2nd Int. Energy Conversion Eng. Conference, Providence, Rhode Island, AIAA2004-5583, 2004.

[25] S. Wilson, R. Dyson, R. Tew and M. Ibrahim, "Multi-D CFD Modeling of a Free-Piston Stirling 
Convertor at NASA GRC," in 2nd Int. Energy Conversion Eng. Conference, Providence, Rhode Island, AIAA-2004-5573, 2004.

[26] K. Mahkamov and D. Ingham, "Theoretical Investigations of the Stirling Engine Working Processes," in AIAA-2000-2815, 2000.

[27] K. Mahkamov, "An Axisymmetric Computational Fluid Dynamics Approach to the Analysis of the Working Process of a Solar Stirling Engine," J. of Solar Energy Eng., vol. 128, pp. 45-53, 2006.

[28] M. Ibrahim, M. Mittal, N. Jiang and T. Simon, "Validation of Multi-Dimensional Stirling Engine Codes: Modeling of the Heater Head," in 3rd International Energy Conversion Engineering Conference, San Francisco, California, AIAA 2005-5654, 2005.

[29] K. Mahkmov, "Design Improvements to a Biomass Stirling Engine Using Mathematical Analysis and 3D CFD Modeling," J. of Energy Resources Technology, vol. 128, pp. 203-215, 2006.

[30] W. Chen, K. Wong and Y. Chang, "A Computational Fluid Dynamics Study on the Heat Transfer Characteristics of the Working Cycle of a Low-Temperature-Differential $\gamma$-Type Stirling Engine," Int. Journal of Heat and Mass Transfer, vol. 75, pp. 145-155, 2014.

[31] S. Alfarawi, R. AL-Dadah and S. Mahmoud, "Influence of Phase Angle and Dead Volume on Gamma-Type Stirling Engine Power Using CFD Simulation," Energy Conversion and Management, vol. 124, pp. 130-140, 2016.

[32] S. El-Ghafour, "Design and Computational Fluid Dynamics Simulation of a Solar Stirling Engine," Ph.D. Dissertation, University of Port Said, Port Said, Egypt, 2018.

[33] "ANSYS FLUENT Theory Guide," Release 17.0, ANSYS, Inc., 2016.

[34] J. Tu, G. Yeoh and G. Liu, Computational Fluid Dynamics: A Practical Approach, Elsevier Inc., Oxford, UK, 2008.

[35] S. Costa, I. Barreno, M. Tutar, J. Esnaola and H. Barrutia, "The Thermal Non-Equilibrium Porous Media Modelling for CFD Study of Woven Wire Matrix of a Stirling Regenerator," Energy Conversion and Management, vol. 89, pp. 473-

\section{محاكاة بأستخدام ديناميكا الموائع الحسابية و تحليل المفاقيد لمحرك ستيرلتج من التوع بيتا}

يتم فى هذا البحث عمل محاكاة ثلاثثية الأبعاد بأستخدام ديناميكا الموائع الحسابية لمحرك ستيرلنج من النوع بيتا. اولا يتم عمل توصيف كامل لمجال

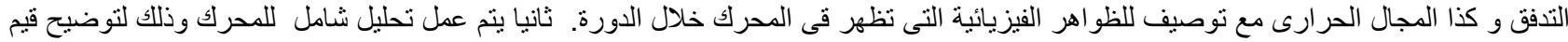

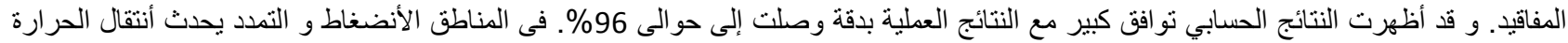

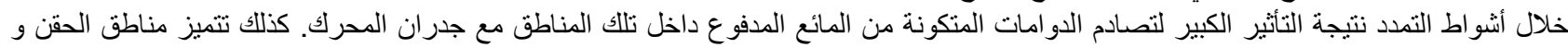

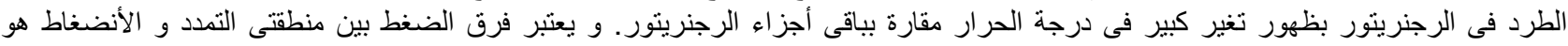

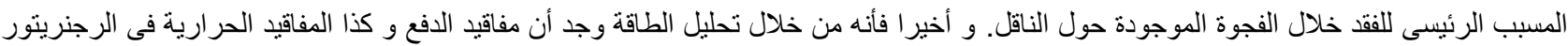

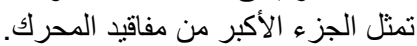

